OPEN ACCESS

Edited by:

Sukhjiwan Kaur,

Department of Economic

Development Jobs Transport and

Resources, Australia

Reviewed by:

Junsong Pan,

Shanghai Jiao Tong University, China Prabhakaran Sambasivam,

Griffith University, Australia

*Correspondence:

Rebecca Grumet grumet@msu.edu

Specialty section: This article was submitted to

Plant Breeding,

a section of the journal

Frontiers in Plant Science

Received: 07 August 2019 Accepted: 04 November 2019

Published: 27 November 2019

Citation:

Rett-Cadman S, Colle M, Mansfeld B, Barry CS, Wang Y, Weng Y, Gao L, Fei $Z$ and Grumet $R$ (2019) QTL and Transcriptomic Analyses Implicate Cuticle Transcription Factor SHINE as a Source of Natural Variation for Epidermal Traits in Cucumber Fruit.

Front. Plant Sci. 10:1536.

doi: 10.3389/fpls.2019.01536

\section{QTL and Transcriptomic Analyses Implicate Cuticle Transcription Factor SHINE as a Source of Natural Variation for Epidermal Traits in Cucumber Fruit}

\author{
Stephanie Rett-Cadman ${ }^{1}$, Marivi Colle ${ }^{1}$, Ben Mansfeld ${ }^{1}$, Cornelius S. Barry ${ }^{1}$, \\ Yuhui Wang ${ }^{2,3}$, Yiqun Weng ${ }^{2,3}$, Lei Gao ${ }^{4}$, Zhangjun Fei ${ }^{4}$ and Rebecca Grumet ${ }^{1 *}$ \\ ${ }^{1}$ Department of Horticulture and Graduate Program in Plant Breeding, Genetics and Biotechnology, Michigan State \\ University, East Lansing, MI, United States, ${ }^{2}$ Department of Horticulture, University of Wisconsin, Madison, WI, United \\ States, ${ }^{3}$ USDA-ARS, Vegetable Crops Research Unit, Madison, WI, United States, ${ }^{4}$ Boyce Thompson Institute, Cornell \\ University, Ithaca, NY, United States
}

The fruit surface is a unique tissue with multiple roles influencing fruit development, post-harvest storage and quality, and consumer acceptability. Serving as the first line of protection against herbivores, pathogens, and abiotic stress, the surface can vary markedly among species, cultivars within species, and developmental stage. In this study we explore developmental changes and natural variation of cucumber (Cucumis sativus L.) fruit surface properties using two cucumber lines which vary greatly for these traits and for which draft genomes and a single nucleotide polymorphism (SNP) array are available: Chinese fresh market type, Chinese Long '9930' (CL9930), and pickling type, 'Gy14'. Thin-section samples were prepared from the mid-region of fruit harvested at 0, 4, 8, 12, 16, 20, 24 and 30 days post pollination (dpp), stained with Sudan IV and evaluated for cuticle thickness, depth of wax intercalation between epidermal cells, epidermal cell size and shape, and number and size of lipid droplets. 'Gy 14 ' is characterized by columnar shaped epidermal cells, a 2-3 fold thicker cuticular layer than CL9930, increased cuticular intercalations between cells and a larger number and larger sized lipid droplets. In both lines maximal deposition of cuticle and increase in epidermal size coincided with exponential fruit growth and was largely completed by approximately $16 \mathrm{dpp}$. Phenotyping and quantitative trait locus mapping (QTL) of fruit sampled from an $\mathrm{F}_{7}: \mathrm{F}_{8}$ Gy14 $\times$ CL9930 recombinant inbred line (RIL) population identified QTL regions on chromosomes 1, 4 and 5. Strong QTL for epidermal cell height, cuticle thickness, intercalation depth, and diameter of lipid droplets co-localized on chromosome 1. SSR markers on chromosome 1 were used to screen for recombinants in an extended RIL population to refine the QTL region. Further fine mapping by KASP assay combined with gene expression profiling suggested a small number of candidate genes. Tissue specificity, developmental analysis of expression, allelic diversity and gene function implicate the regulatory factor CsSHINE1/ WIN1 as a source of natural variation for cucumber fruit epidermal traits.

Keywords: cucumber, Cucumis sativus, fruit surface, cuticle, lipid droplet, epidermis, fruit development 


\section{INTRODUCTION}

Fruit surfaces playimportant roles in fruit development, maturation, and post-harvest quality. During growth and maturation, the exocarp is the first line of defense against herbivores, pathogens, and abiotic stresses such as dehydration, UV irradiation, and mechanical pressure. Following harvest, morphological features of the fruit surface can influence consumer preference and fruit quality. The epidermal cell structure can influence fruit firmness and susceptibility to damage. Cuticle structure and waxiness can modify external appearance such as glossiness and uniformity, and modulate rate of evaporative water loss, susceptibility to cracking and pathogen infection, and material penetration into the fruit surface (reviews: Hen-Avivi et al., 2014; Lara et al., 2014; Martin and Rose, 2014). These factors, in turn, influence handling practices in the commercial market chain.

For cucumber (Cucumis sativus), different market types vary substantially with respect to fruit surface features that influence consumer preferences, suitability for shipping, handling and storage, or performance demands for processing (pickling). Cucumber market types can vary considerably with regard to post-harvest longevity. Weight loss during the market chain is a primary concern and is influenced by epidermal properties including skin toughness and waxiness (Patel and Panigrahi, 2019; https://www.postharvest.net.au/product-guides/cucumber/). Wax load is inversely related with rate of water loss (Wang et al., 2015a), and untreated cucumber fruit can have a shelf life of less than a week (Patel and Panigrahi, 2019). As a result, packaging methods for fresh market cucumbers include wrapping in plastic, which is both expensive and environmentally undesirable, or treating the fruit with edible coatings.

The importance of the cuticle in product quality of fleshy fruits, coupled with relative ease of isolation from certain species, has driven studies focused on the biosynthesis and properties of fruit cuticles (Hen-Avivi et al., 2014; Lara et al., 2014; Martin and Rose, 2014). While much of the cuticle biosynthetic pathway has been established in Arabidopsis, characterization of mutants with altered cuticle composition (Isaacson et al., 2009; Nadakuduti et al., 2012; Yeats et al., 2012; Petit et al., 2014), tissue-specific transcriptomic analysis of developing tomato fruit peel (MintzOron et al., 2008; Matas et al., 2011), and QTL mapping of introgression lines of wild tomato species (Solanum pennelli, Solanum habroachaites) (Cohen et al., 2017; Fernandez-Moreno et al., 2017) also have identified numerous genes associated with fruit cuticle development and composition. In cucumber, homologs of two key cuticle biosynthetic enzyme genes involved in cutin and wax biosynthesis, eceriferum (CER1) and WAX2, have been cloned (Wang et al., 2015b). Decreased expression of CER1 and WAX2 was associated with reduced wax load and increased water loss from harvested fruits.

Deposition of the cuticle and epicuticular waxes is developmentally programmed during organ growth to accommodate coverage required by increased surface area (Martin and Rose, 2014; Ingram and Nawrath, 2017). The precursors needed for cuticle and wax deposition are produced by epidermal cells and delivered to the fruit surface (Kunst and Samuels, 2003; Matas et al., 2010; Matas et al., 2011; Yeats and
Rose, 2013; Huang 2018). Accordingly, CsCER1 and CsWAX2 are preferentially expressed in cucumber epidermal tissue (Ando et al., 2012; Wang et al., 2015a, b). For many species, cuticle deposition ceases during early fruit development, often before the fruit has reached maximum size and prior to the onset of ripening (Lara et al., 2014). We have observed that cuticle thickness in the pickling cucumber cultivar, 'Vlaspik', increases dramatically during the rapid growth phase from 4 to 16 days post-pollination (dpp) (Ando et al., 2012; Ando et al., 2015). The time period of $8-12 \mathrm{dpp}$ also was marked by peak expression of genes associated with cuticle biosynthesis, such as several extracellular GDSL motif lipase/hydrolase proteins and lipid transfer proteins which have been implicated in lipid transport to extracellular surface (Ando et al., 2012).

Cuticle-related transcription factors have been identified from Arabidopsis, including the AP2 domain superfamily member, shine1 (SHN1), or win1 (WAX INDUCER1) (Aharoni et al., 2004; Broun et al., 2004). In tomato fruit, an exocarp-expressed SHN clade member, SlSHN3, regulates cuticle production; suppression of SlSHN3 reduced cuticle production and caused a glossier fruit surface (Shi et al., 2013). In cucumber fruit, a preferentially peelexpressed homolog of SHN1 (CsaV31g030200) exhibited peak transcription at 8-12 dpp, in concert with expression of the suite of cuticle biosynthesis associated genes (Ando et al., 2012). Several other transcription factors identified in tomato including MYB, MADS and homeodomain leucine zipper family members are also associated with regulation of production of cutin and wax components and cutin-localized secondary metabolites such as flavonols and terpenes (Adato et al., 2009; Isaacson et al., 2009; Gimenez et al., 2015). Many of the cuticle related transcription factors, including AtSHN1/SlSHN3, are also linked to epidermal cell patterning. In Arabidopsis and tomato MIXTAlike and leucine zipper transcription factors regulate both cuticle production and epidermal cell formation (Nadakuduti et al., 2012; Oshima et al., 2013; Lashbrooke et al., 2015a), and in maize, the glossy trait conferred by the AP2/EREBP transcription factor gene, GL15, influences epicuticular wax deposition, leaf hair formation, and cell shape (Moose and Sisco, 1996; Lauter et al., 2005).

While much of our understanding of epidermal cell structure and cuticle development has been derived from mutant or overexpression analyses using a limited number of model systems, little is known about the factors driving variation in natural populations (Petit et al., 2017). Current genetic resources and genomic tools can greatly facilitate our ability to identify and utilize sources of natural diversity across an increasing number of species, including cucumber. Reference genomes have been developed for representatives of two morphologically distinct cucumber market classes: the fresh market Chinese Long type, 'CL9930', and the American pickling type, 'Gy14' (Huang et al., 2009; Yang et al., 2012; Wang et al., 2018). In addition to obvious differences in fruit size and shape, CL9930 and Gy14 show markedly different epidermal and cuticle structures including amount and location of cuticle and wax deposition, number and size of lipid droplets present in epidermal cells, and size and shape of epidermal cells (Colle, 2015). In this study we sought to characterize epidermal cell growth and cuticle and wax 
deposition during cucumber fruit development, and identify genomic regions and candidate genes associated with variation using recombinant inbred lines (RILs) derived from progeny of Gy14 × CL9930. Several QTL were identified, including a major QTL on chromosome 1 associated with cuticle thickness, epidermal cell height, intercalation depth, and diameter of lipid droplets. Combined fine mapping, transcriptional analysis, and allelic diversity among cucumber accessions implicated the transcription factor CsSHINE1/WIN1 as a regulator of natural variation for cucumber fruit epidermal traits.

\section{MATERIALS AND METHODS}

\section{Plant Materials and Growth Conditions}

Plant Materials. Seed of cucumber (C. sativus L.) lines Gy14 (American pickling cucumber inbred line) and CL9930 (Chinese long type) were originally obtained from the University of Wisconsin and multiplied in the greenhouse. Pickling type cultivar Vlaspik was obtained from Seminis Vegetable Seed Inc, Oxnard, CA and American slicing type cultivar Poinsett 76 from Seedway, Hall NY. Our prior studies show that despite differences in size and shape, all four varieties exhibit a typical developmental pattern for cucumber with a period of cell division ( $\sim-4 \mathrm{dpp})$, followed by exponential growth, and approaching full size at 16-20 dpp (Ando et al., 2012; Colle, 2015; Colle et al., 2017). The three American varieties all have thick cuticles, while CL9930 has a thin cuticle (Supplementary Table 1)

Developmental Study. Gy14 and CL9930 plants were grown in the greenhouse (Michigan State University Plant Science Greenhouse Complex, East Lansing MI) in summer 2017 in $4 \mathrm{~L}$ plastic pots with Suremix Perlite soil medium (Michigan Grower Product, Inc., Galesburg, MI). The plants were watered and fertilized twice daily (with $44 \mathrm{ppm}$ nitrogen of Peters Professional 20-20-20 General Purpose; Scotts, Marysville, $\mathrm{OH}$ ) using an automated drip irrigation system (Dositron model D14MZ2, Clearwater FL). Supplemental high pressure sodium lights were used to provide a $16-\mathrm{h}$ photoperiod. Pest and disease control were performed according to standard management practices in the greenhouse. When the plants initiated female flower production, a single ovary from each of 48 plants per line were hand-pollinated on the same day to ensure comparable environmental conditions for all fruit during development and provide sufficient fruit for each harvest date. Only one fruit was set per plant to have consistent developmental rates for all fruits by preventing competition for resources among fruits. At each sample date $(0,4,8,12,16,20,24$, and $30 \mathrm{dpp})$ three fruits per line (biological replicates)/age were harvested. Three samples (technical replicates) derived from the midsection of each fruit were prepared for microscopy.

RIL Analyses. A Gy14 × CL9930-derived $\mathrm{F}_{7: 8}$ RIL population (Weng et al., 2015) that was previously genotyped by SNP array (Rubenstein et al., 2015) was grown in the greenhouse in Fall 2016, as described above. Each of 110 RILs and both parental lines were grown in triplicate (biological replicates) in a randomized, complete block design. Ovaries were hand pollinated and only one fruit per plant was set to minimize inter-fruit competition.
Fruits were harvested at $16 \mathrm{dpp}$ and prepared as described below. An extended $\mathrm{F}_{7: 8}$ RIL population, consisting of 375 lines, was screened using SSR markers to identify recombinants for regions in chromosomes 1 and 4. Recombinant lines, parents, and reciprocal $\mathrm{F}_{1} \mathrm{~s}$ were grown in triplicate (biological replicates) in the field in Summer 2018, in a randomized, complete block design at the Michigan State University Horticulture Teaching and Research Center, East Lansing, MI. Bee-pollinated flowers were tagged at anthesis. Fruit were harvested at 20-22 dpp and two samples (technical replicates) derived from the midsection of each fruit were prepared for microscopy as described below. Pest and disease control were performed according to standard management practices under field conditions. A subset of 17 RILs recombinant in the region of interest on chromosome 1 along with both parents were grown in the greenhouse in Spring 2019 under conditions described above to provide replication in different environments. Fruit were harvested at 16-20 dpp.

RNA-Seq Experiment. Fruit from the cultivars 'Poinsett 76 ' and 'Gy 14' were grown under greenhouse conditions as described above. Flowers were hand pollinated, such that 8 and $16 \mathrm{dpp}$ fruit were harvested on the same day. Peels from three fruit (biological replicates) were harvested for each age and genotype and immediately frozen in liquid nitrogen and stored at $-80^{\circ} \mathrm{C}$ until RNA extraction.

CsSHN1 expression analysis. Sixty plants of CL9930, Gy14, and Vlaspik were grown under greenhouse conditions as described above with the following modifications: supplemental lights provided an 18-h light cycle and plants were hand fertilized once a week. One or two flowers from the third to fifth node were hand-pollinated on the same day on each plant for each genotype; a single fruit was allowed to develop. Three fruits each from CL9930, Gy14 and Vlaspik were collected at anthesis, 4, 8, 12,16 , and $20 \mathrm{dpp}$.

\section{Microscopy and Measurement of Epidermal Traits}

A wedge $\left(\sim 1 \mathrm{~cm}^{3}\right)$ was cut from the mid-section of each fruit and sliced to $\sim 0.1 \mathrm{~mm}$ thickness by a sliding block microtome. All methods pertaining to staining with Sudan IV (SigmaAldrich, St. Louis MO) and subsequent washing were performed according to the methods Buda et al. (2009) with the exception of RIL experiments (Summer 2018 and Spring 2019) when samples were mounted in glycerin (Columbus Chemical Industries, Columbus WI) instead of distilled water. All samples in water were imaged by microscopy the same day; glycerin mounted samples were imaged within one week. Images for the RIL population from the Fall 2016 experiment were captured using an EVOS FL Auto imaging system (ThermoFisher Scientific; http://www.thermofisher.com) with $400 \times$ magnification and analyzed using the Nikon NIS-Elements BR imaging system. For the developmental study (Summer 2017) and the extended RIL population (Summer 2018, Spring 2019), images were obtained using a Nikon Eclipse Ni-U microscope and Nikon DS-Fi3 camera (Nikon Instruments Inc.; Melville, NY) at 600× and 200× magnification, respectively. Epidermal features were measured as shown in Figure 1. To allow for better comparison among samples 


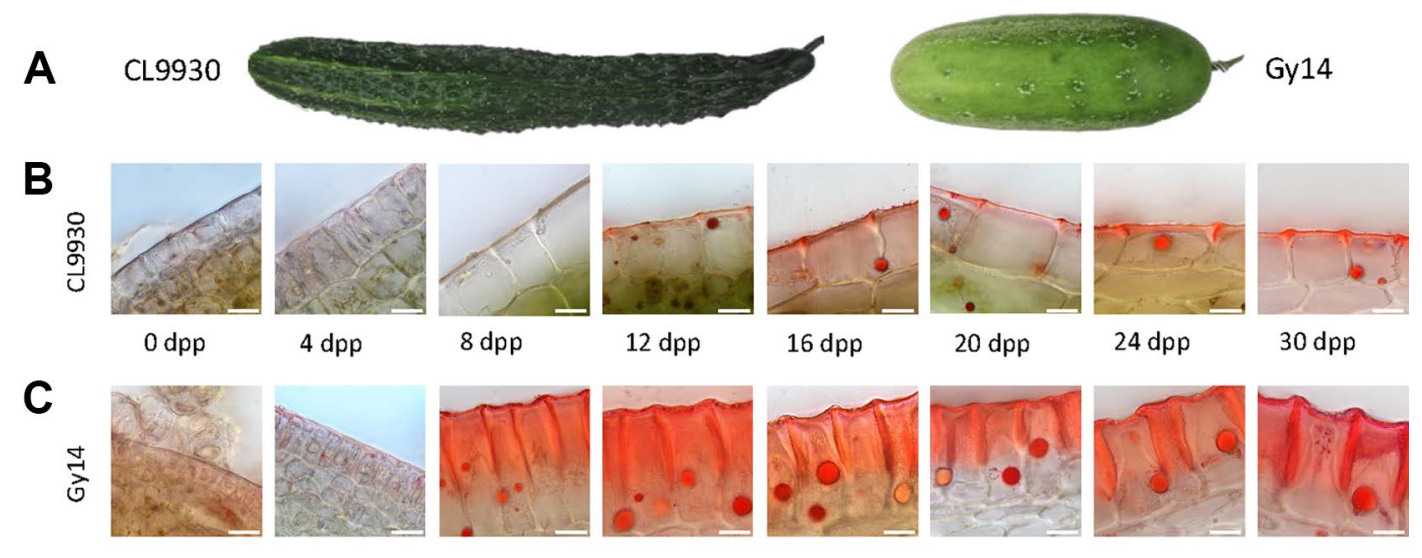

D
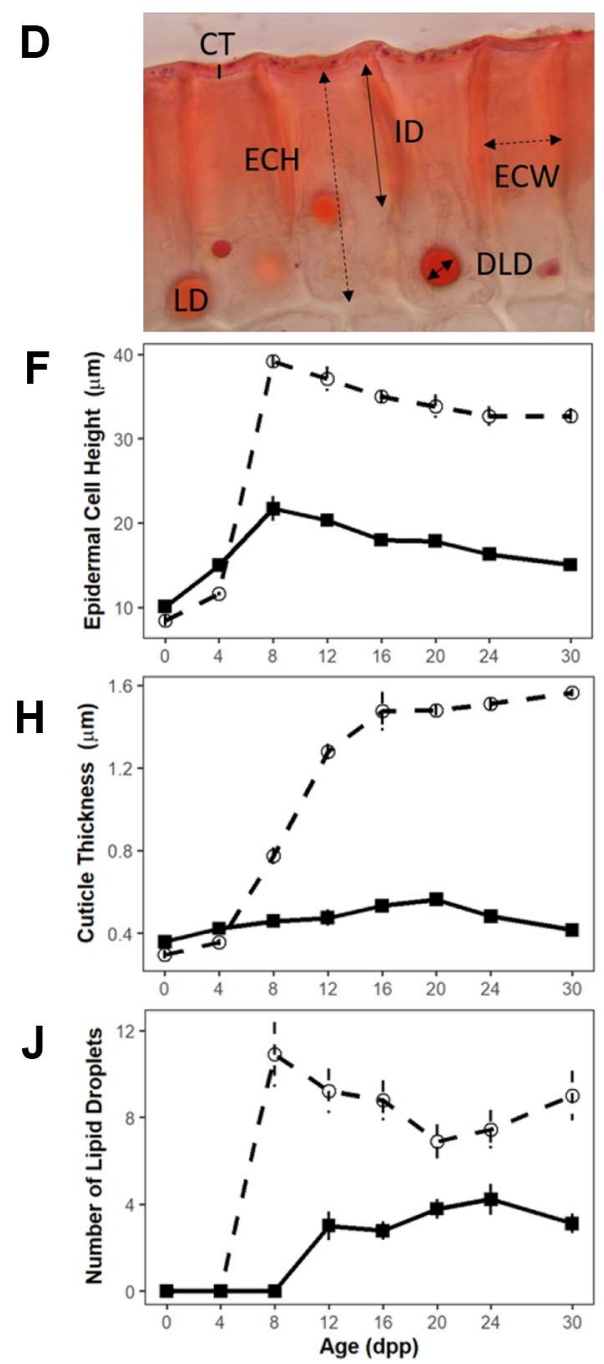

E

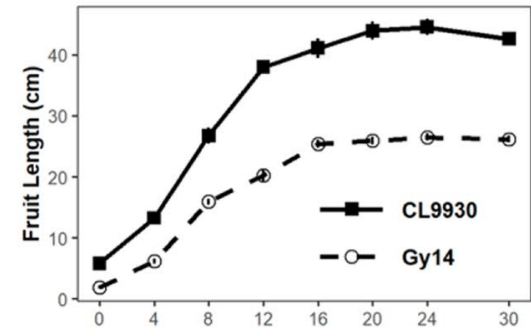

G
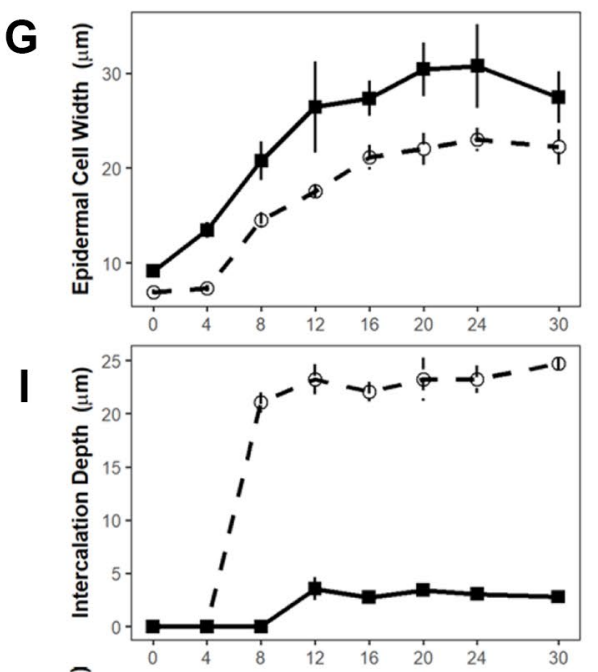

K

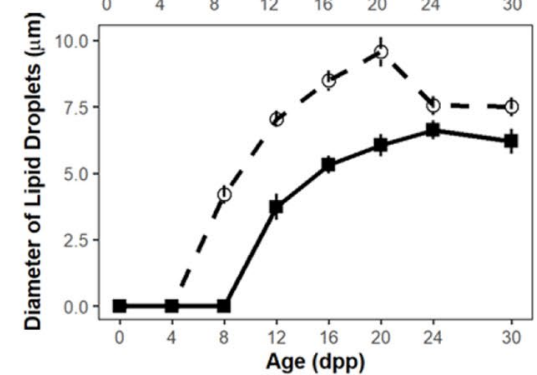

FIGURE 1 | Developmental study of cucumber fruit epidermal traits. (A) CL9930 and Gy14 fruit at 16 days post pollination (dpp). (B, C) Cross sections of CL9930 (B) and Gy14 (C) at 0, 4, 8, 12, 16, 20, 24, and 30 days post pollination (dpp). Magnification =1,000x. Scale bar $=10 \mu \mathrm{m}$. Samples were taken from the midsection of the fruit. (D) Cross section illustrating traits measured; lipids were stained with Sudan IV. Cuticle thickness (CT) is represented by a solid, vertical line; Epidermal cell height (radial dimension, ECH) and Epidermal cell width (ECW) by dashed, double-headed arrows; Intercalation depth (ID) by a solid, double-headed arrow; LD indicates lipid droplet, and lipid droplet diameter (LDD) is represented by a solid, double-headed arrow. (E) Fruit length for CL9930 (solid line) and Gy14 (dotted line). (F-K) Developmental progression of fruit epidermal traits for CL9930 (solid line) and Gy14 (dotted line): (F) epidermal cell height (radial dimension), (G) epidermal cell width $\mathbf{( H )}$ cuticle thickness, (I) intercalation depth, $\mathbf{(} \mathbf{(})$ number of lipid droplets in $120 \mu \mathrm{m}$ linear region of epidermal cells, (K) diameter of lipid droplets. Each value is the mean of 3 replicate fruits \pm S.E. 
and avoid influence of warts and spines, all measurements were made in areas between spines. To standardize measurements of epidermal features, a line of $120 \mu \mathrm{m}$ (developmental study) or $450 \mu \mathrm{m}$ (RIL populations) was drawn across a given sample and features were measured within that area. Three measurements across the sample were taken for cuticle thickness (CT), intercalation depth (ID), and epidermal cell height (ECH); the mean value was used in subsequent analyses. Epidermal cell width (ECW) was determined by dividing $450 \mu \mathrm{m}$ by the number of epidermal cells in that area. The number of lipid droplets (NLD) were counted in the given area. The diameters of all lipid droplets (DLD) in this area were measured and the average used in subsequent analyses. Calculation of Pearson's correlation coefficients among traits were conducted using the $\mathrm{R}$ package 'GGally' (https://github.com/ggobi/ggally).

\section{Mapping of Epidermal Traits QTL Analyses}

For QTL analysis, a subset of 916 unique markers were used from a previously constructed genetic map (Rubenstein et al., 2015; Weng et al., 2015). Composite interval mapping (CIM) was performed with QTL Cartographer v2.5 using the standard model Zmapqtl 6 with walking speed of $1 \mathrm{cM}, 5$ background markers, and window size of $5 \mathrm{~cm}$ (Wang et al., 2012). The forward and backward method was used to select markers as cofactors. The LOD significance threshold was determined by a 1,000-permutation test at 5\% probability.

\section{SSR Screening}

Microsatellite (SSR) markers within the 2.0-LOD intervals at the two QTL loci on Chr1 and Chr4 were used to genotype an expanded population of $375 \mathrm{~F}_{7: 8}$ Gy14× 9930 RILs to identify recombinants between the flanking markers. Due to the inconsistency in physical locations of the flanking SNP markers in 9930 v2.0 and Gy14 v2.0 draft genome assemblies, multiple SSR markers in the two target regions were employed. Information of markers used to identify recombinants is provided in Supplementary Table 2. DNA extraction, PCR amplification of molecular markers and gel electrophoreses followed Gao et al. (2016).

\section{KASPTM Screening}

DNA Isolation and Quantification. Tissue samples ( 50 mg) from young leaf tissue of cucumber seedlings ( $\sim 1-2$ weeks) were lyophilized in a freeze-dryer and ground into fine powder with a high-throughput homogenizer (OPS Diagnostics, Lebanon, NJ). DNA was isolated and quantified as described in Wiersma et al., 2017. Briefly, DNA was isolated using the Mag-Bind ${ }^{\circledR}$ Plant DNA Plus 96 Kit (M1128, Omega Bio-Tek,Norcross, GA) on a King Fisher Flex Purification System (Thermo Scientific, Waltham, MA). DNA was quantified using the Quant-iT ${ }^{\mathrm{TM}}$ PicoGreen ${ }^{\circledR}$ dsDNA Kit (Life Technologies Corp., Grand Island, NY) on a CFX384 Real-Time thermal cycler, C1000 (BioRad, Hercules, CA).

SNP Calling and KASP ${ }^{\mathrm{TM}}$ Assay. From the expanded RIL population, 87 lines selected to be recombinants in QTL regions 1 or 4 were grown in triplicate in field conditions and phenotyped as described above. Due to the strength of the QTL on chromosome 1, further fine mapping was performed using the 29 lines that were identified by SSR assay to be recombinants in that region. The draft genomes of Gy14 (Version 2, cucurbitgenomics.org) and CL9930 (Version 3, cucurbitgenomics.org) were aligned using the nucmer function of MUMmer 4 (Marçais et al., 2018). Single nucleotide polymorphisms (SNPs) were then called using the show-snps function. SNPs immediately flanking the region of interest on chromosome 1 and at an interval of $0.25 \mathrm{Mb}$ along this region were used to design allele specific forward $\mathrm{KASP}^{\mathrm{TM}}$ (LGC, Teddington, Middlesex, UK) and common reverse primers, where all CL9930 alleles would express a FAM signal, while the Gy14 allele would express a HEX signal. $\mathrm{KASP}^{\mathrm{TM}}$ markers that met the following criteria were selected for use in assay: GC content of 30-55\%; approximate melting temperature of $64 \pm 2{ }^{\circ} \mathrm{C}$; length of 21-28 bp; product size of 50-100 bp; limited to no secondary structure or repeats; and GC clamp with no more than 3 Gs or Cs in the last 5 bp of the primer (Supplementary Table 3). PCR thermocycling and fluorescence detection was conducted using a CFX384 Real-Time thermal cycler (BioRad), where alleles were determined using the CFX manager software (v.3.1).

\section{Expression Analyses RNA-Seq Experiment}

Sample Collection and RNA Extraction. Peels were collected from 8 and 16 dpp of 'Poinsett 76' and 'Gy 14' using a vegetable peeler, immediately frozen in liquid nitrogen and stored at $-80^{\circ} \mathrm{C}$ until further use. Three fruit (biological replicates) were collected for each age and genotype. Peel samples were ground in liquid nitrogen using a mortar and pestle. RNA extraction was performed using the MagMAX Plant RNA Isolation Kit protocol (Thermo Fisher Scientific, Waltham MA) with the exception of increased amount of tissue and buffer; approximately 100-150 mg tissue was transferred to a $1.5 \mathrm{ml}$ tube with $1,000 \mu \mathrm{l}$ of lysis buffer. After lysis and centrifugation as per the protocol, supernatant was transferred to a 96-deep-well plate for highthroughput RNA extraction, on a KingFisher Flex Purification System. Immediately after the run was complete, the 96-well plate was transferred to storage at $-80^{\circ} \mathrm{C}$. RNA concentration and quality were measured using Qubit 2.0 Fluorometer (Thermo Fisher Scientific) and LabChip GX (Perkin Elmer, Waltha MA), respectively. All samples had a minimum RNA quality score of 8 .

RNA-Seq Library Preparation and Sequencing. RNA-seq libraries were prepared at Michigan State University's Research Technology Support Facility, using the Illumina TruSeq Stranded mRNA Library Preparation Kit on a Sciclone G3 workstation following manufacturer's recommendations. An additional cleanup with $0.8 \times$ AmpureXP magnetic beads was performed after completion of library preparation. Quality control and quantification of completed libraries was performed using a combination of Qubit dsDNA HS and Advanced Analytical Fragment Analyzer High Sensitivity DNA assays. The libraries were divided into two pools of 15 libraries each. Pools were quantified using the Kapa Biosystems Illumina Library 
Quantification qPCR kit. Each pool was loaded onto one lane of an Illumina HiSeq 4000 flow cell and sequencing was performed in a $1 \times 50$ bp single read format using HiSeq 4000 SBS reagents. Base calling was done by Illumina Real Time Analysis (RTA) v2.7.7 and output of RTA was demultiplexed and converted to FastQ format with Illumina Bcl2fastq v2.19.1.

Differential Expression Analysis. Reads were cleaned, and adaptor sequences were removed using Trimmomatic v. 0.34 (Bolger et al., 2014) with the following settings: LEADING:3 TRAILING:3 SLIDINGWINDOW:4:15 MINLEN:35. Quality control was performed using FastQC (http://www.bioinformatics. bbsrc.ac.uk/projects/fastqc). A cucumber transcriptome fasta file was made from the 'Chinese Long' (v2) (Huang et al., 2009; Li et al., 2011) genome using the gffread function from the cufflinks software package (Trapnell et al., 2010) and high-quality reads were then quasi-mapped to the transcriptome using Salmon v. 0.9.1 (Patro et al., 2017) with default settings.

Read quantification data was imported into $\mathrm{R}$ using the tximport R package (Soneson et al., 2015) and differential expression analysis was performed using DEseq2 (Love et al., 2014) with log-fold-change-shrinkage. Age and genotype were combined into a single factor for differential expression analysis and contrasts between the four conditions ('Poinsett 76' $8 \mathrm{dpp}$, 'Poinsett 76' 16 dpp, 'Gy14' 8 dpp, 'Gy14’ 16 dpp) were performed. Differentially expressed genes were called significant using an adjusted p-value (Benjamini and Hochberg, 1995) of less than 0.05. A cutoff expression change of above two-fold was used to define biological significance. Expression data for candidate genes from CL9930 was accessed using the gene expression profiles function (Zheng et al., 2019) of http://cucurbitgenomics. org/; gene expression project PRJNA 312872 (Wei et al., 2016).

\section{Expression Analysis of CsSHN1 During Fruit Development}

Pericarp samples isolated from the middle part of the fruit of CL9930, Gy14 and Vlaspik were immediately frozen in liquid nitrogen. Total RNA samples from the pericarp tissue were prepared using the Trizol method (Thermo Fisher Scientific), followed by DNase I treatmentand clean up (Qiagen, Germantown $\mathrm{MD}$ ). The amount of RNA for each sample was measured using the nanodrop ND-1000 (Thermo Fisher Scientific). First strand cDNA synthesis was performed using the High Capacity RNAto-cDNA Kit (Thermo Fisher Scientific) and by following the protocol described by Ando and Grumet (2010). Gene-specific primers were designed using Primer Express software (Applied Biosystems, Forest City CA). The ABI Prism 7900HT Sequence Detection System was used for qRT-PCR analysis. Revolution PCR Master Mix (Integrated Scientific Solutions, San Diego CA) with ROX as reference dye was used for gene amplification. C. sativus polyubiquitin (CuSa200910_13711) was used as an endogenous control for normalization. Expression of target genes was assessed with reference to corresponding standard curves. qRT-PCR was performed using cDNA of three fruits (three biological replicates)/genotype with three technical replicates/biological replicate. Data were analyzed by analysis of variance (ANOVA) and Tukey HSD protocol in SAS (SAS Institute, Cary, NC).

\section{Analysis of CsSHN1 Alleles}

Identification of CsSHN1 Alleles in Gy14, Poinsett 76 and Vlaspik. DNA was extracted from three lines 'Gy 14,' 'Poinsett 76', and 'Vlaspik' using the Kingfisher DNA extraction robot as described in Wang et al. (2018). After quantitation, all libraries were pooled in equimolar amounts which was loaded on one lane of an Illumina HiSeq 2500 High Output flow cell (v2) alongside other samples with a targeted coverage of $\sim 30 \times$. Sequencing was carried out using HiSeq SBS reagents in a $2 \times 150$ bp paired end format (PE150). Reads were cleaned and adaptor sequences were removed using Trimmomatic v. 0.33 (Bolger et al., 2014). Reads were mapped to the 'Chinese Long 9930' (v2) (Huang et al., 2009; Li et al., 2011) cucumber genome using BWA-MEM (Li and Durbin, 2009). Duplicate reads were marked with Picard (https://broadinstitute.github.io/picard/) and the GATK "Best Practices" pipeline was used for variant calling (McKenna et al., 2010; DePristo et al., 2011; Van der Auwera et al., 2013). Variants were hard-filtered with the GATK base recommendations. Initial analyses were done with CL9930v2 but nucleotide positions were later converted to CL9930v3.

Survey of Cucumber Germplasm for CsSHN1 Alleles. Cucumber accessions for which resequencing data were available were examined for the nucleotide present at position 16961026 within the CsSHN1 locus (CsaV3_1G030200). Sequence data for 115 accessions were available from Qi et al. (2013). Data for an additional 89 accessions (Supplementary Table 4), comprising a portion of the cucumber core outlined in Wang et al., 2018, were also analyzed. Samples were included for which there were at least 10 reads at position 16961026.

\section{RESULTS}

\section{Developmental Progression of Fruit Epidermal Traits}

The parental inbred lines Gy14 (a pickling breeding line) and CL9930 (an Asian fresh market breeding line) differ for fruit size, shape, and epidermal properties (Figure 1). Epidermal cells of Gy14 have a palisade orientation, thicker cuticle, and deeper cuticular intercalations between cells, whereas CL9930 has a flatter epidermal cell shape, with wider cells, thinner cuticle and minimal cuticular intercalation. An additional striking feature of the epidermal cells was the presence of large circular droplets brightly stained with the red lipid-soluble dye, Sudan IV. The number and size of the lipid droplets also differed between the two lines of interest, with larger and more numerous lipid droplets in Gy14.

A developmental study was performed in the greenhouse to assess changes in epidermal properties during fruit growth and maturation. To minimize effects of competition on growth rate, a single fruit was set per plant. Fruit were harvested at 0 (anthesis), 4, 8, 12, 16, 20, 24, and $30 \mathrm{dpp}$ (maturity). Subsequent to initial fruit set and the period of active cell division (0-4 dpp) (Fu et al., 2010; Ando et al., 2012; Colle et al., 2017), fruit epidermal properties changed dramatically, especially in Gy14 (Figures 1F-K). Increases in epidermal cell height and width, cuticle thickness and intercalation between epidermal cells, and 
lipid droplet number and size, generally showed a sigmoidal trend with fruit age. The greatest increases for most traits occurred between 4 and $12 \mathrm{dpp}$, coinciding with the period of exponential fruit growth (Figure 1E). Differences between Gy14 and CL9930 became apparent for most traits between 4 and 8 $\mathrm{dpp}$ and were largely stabilized by $16 \mathrm{dpp}$. Obvious intercalations and appearance of lipid droplet were observed sooner in Gy14, at $8 \mathrm{dpp}$, rather than $12 \mathrm{dpp}$ in CL9930.

\section{Fruit Epidermal QTLs}

An $\mathrm{F}_{7: 8}$ Gy14 $\times$ CL9930 RIL population consisting of 110 lines was grown in the greenhouse in 2016 and evaluated for cucumber fruit epidermal traits as described above. Based on the observations of the developmental study, fruit were harvested at $16 \mathrm{dpp}$, after growth had stabilized and differences in fruit epidermal were readily observable. Phenotypic distributions and correlations among the traits are summarized in Figure 2A. Strong, positive correlations were observed among intercalation depth, epidermal cell height, and diameter and number of lipid droplets. Epidermal cell width was negatively correlated with epidermal cell height and number of lipid droplets.

Fourteen QTL were detected on six of the seven cucumber chromosomes (Figure 3 and Table 1). On chromosome 1, a major QTL, ECT1.1 (epidermal cell traits) was detected for cuticle thickness, epidermal cell height, intercalation depth, and diameter of lipid droplets that explained $18.4 \%, 38.1 \%, 44.1 \%$, and $37.9 \%$ of the phenotypic variation for each trait, respectively. A single QTL was found on chromosome 2 for diameter of lipid droplets; chromosome 3 contained one QTL for epidermal cell height; and chromosome 4 had a single QTL for epidermal cell height, intercalation depth, epidermal cell width, and number of lipid droplets. In addition to the QTL for epidermal cell width found on chromosome 4, there also was a QTL detected on chromosome 5. Lastly, several QTL were found on chromosome 6 for intercalation depth, diameter of lipid droplets, and number of lipid droplets. In each case where there were multiple QTL for a single trait, the percent variation explained was greatest for the QTL on chromosome 1.

\section{Marker-Assisted Screening and Fine Mapping of Chromosome 1}

The strongest QTL were detected on chromosome 1, with LOD scores in the range of 6.1-28.7. Linkage analysis also supported the QTL on chromosome 1 (Supplementary Table 5). To narrow the region of interest, SSR markers were designed to flank the peak (at positions 14516668 and 18050191 CL9930 genome v3) with an additional marker in between (position 14783187). These markers were then used to screen an expanded $\mathrm{F}_{7}:{ }_{8} \mathrm{RIL}$ population $(\mathrm{n}=375)$ to identify recombinant individuals in the region of interest. Of these, 87 lines were selected, including 29 identified as recombinant in the designated region on chromosome 1 (Figure 4A).

Selected lines, parents and reciprocal $\mathrm{F}_{1} \mathrm{~s}(\mathrm{~Gy} 14 \times$ CL9930 and CL9930 $\times$ Gy14) were grown in the field in 2018 and phenotyped at 20-22 dpp. With the exception of diameter of lipid droplets, the both $\mathrm{F}_{1}$ 's showed intermediate phenotypes relative to the parents
(Supplementary Figure 1). Similar patterns of distribution and correlations among traits were observed for RILs as for the 2016 experiment in the greenhouse, with the exception of cuticle thickness, likely due to better imaging equipment in 2018 that allowed for more accurate determination of cuticle thickness (Figure 2B). In the 2018 experiment, cuticle thickness was strongly and positively correlated with intercalation depth, diameter of lipid droplets, and epidermal cell height (Figure 2B). The observed correlations among these four traits were consistent with their overlapping QTL positions on chromosome 1.

Combining phenotype data for the four traits with the SSR genotypic data, the region of interest on chromosome 1 was narrowed to approximately $3 \mathrm{Mb}(14.78 \mathrm{Mb}$ to 18.05). Recombinant individuals were then genotyped within this region with a set of seven SNP-based $\mathrm{KASP}^{\mathrm{TM}}$ markers spaced at approximately $0.5 \mathrm{Mb}$ intervals. This narrowed the region of interest to $512 \mathrm{~kb}$, from 16.76 to $17.28 \mathrm{Mb}$ (Figure 4B). A subset of RILs recombinant in this region of chromosome 1 was also grown in the greenhouse in Spring 2019 to test expression of phenotype in different environments. Analysis of data from field Summer 2018 and greenhouse Spring 2019 showed very highly significant correlations (all P values $<2.0 \mathrm{E}-06$ ) between the two conditions for all four traits (cuticle thickness $-r=0.99$; intercalation depth $r=0.96$; diameter of lipid droplets $r=0.84$; epidermal cell height $r=0.86$ ) (Supplementary Table 6).

\section{Candidate Genes Influencing Cucumber Fruit Epidermal Properties}

The $0.51 \mathrm{Mb}$ KASP marker-defined region on chromosome 1 contained 25 annotated genes (CL v3; http://cucurbitgenomics. org/). To refine the list of candidates we performed RNA-seq on fruit peels of the parental lines and further utilized existing expression data from our prior work comparing peels from $8 \mathrm{dpp}$ and 16 dpp Gy14 fruit (Mansfeld et al., 2017) and expression data from Wei et al. (2016) comparing a wide variety of cucumber tissue types in CL9930 (accessed via http://cucurbitgenomics. org/, Gene expression project PRJNA 312872). As we were dealing with fruit epidermal related traits, and based on the developmental analyses showing that increase in cuticle related traits occurred most rapidly between 4 and $12 \mathrm{dpp}$, two criteria were used to filter the genes: (i) preferentially expressed in peel vs. flesh; and (ii) elevated expression at $8 \mathrm{dpp}$ relative to $16 \mathrm{dpp}$. Of the 20 genes in this region showing expression in fruit, four had greater expression in peels than in flesh: CsaV31g030090, a putative heme oxygenase, associated with chlorophyll degradation; CsaV31g030200, a homolog of the cuticle-related transcription factor, SHN1/WIN1; CsaV31g030210, a gene with unknown function; and CsaV31g030360, a glucan endo-1,3-beta glucosidase (Table 2). These genes also showed greater expression in exocarp vs. mesocarp in our prior studies of pickling cucumber cv. Vlaspik, which also has a thick cuticle (Ando et al., 2015). Of the four genes, specificity to the peel was much stronger for CsSHN1, approximately 80-fold vs. 2-fold for CsaV31g030090, CsaV31g030210, and CsaV31g030360. Furthermore, CsSHN1 was essentially exclusively expressed in fruit peel relative to other tissues and organs. In contrast, CsaV31g030090 exhibited 
A
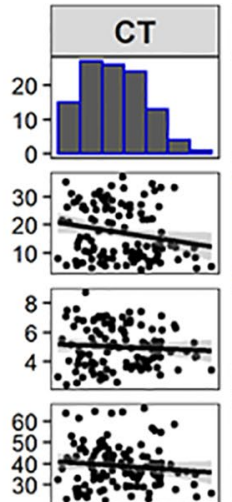

$40-?$
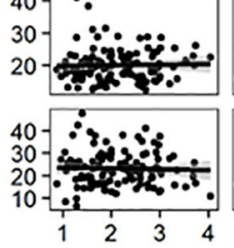

B
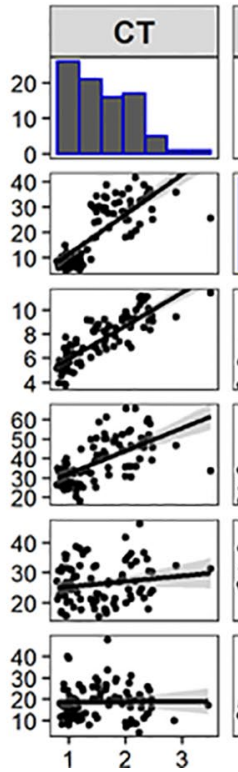
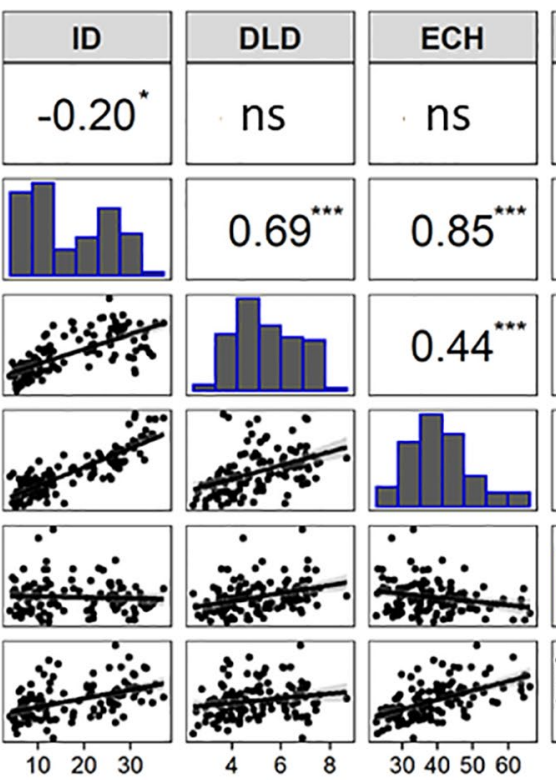

\begin{tabular}{|l|}
\hline ECW \\
\hline $\mathrm{ns}$ \\
\hline
\end{tabular}
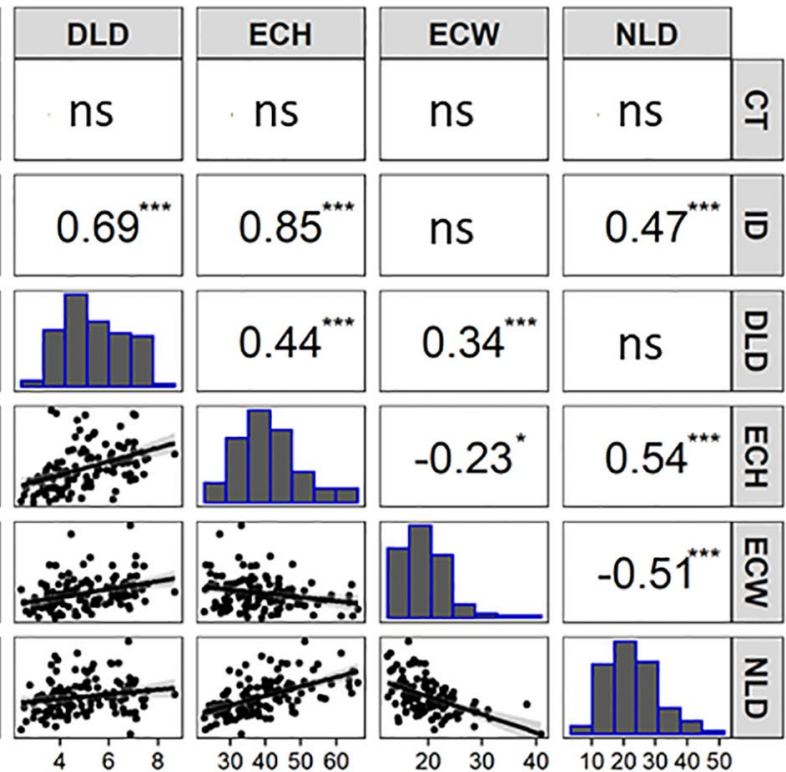

ns
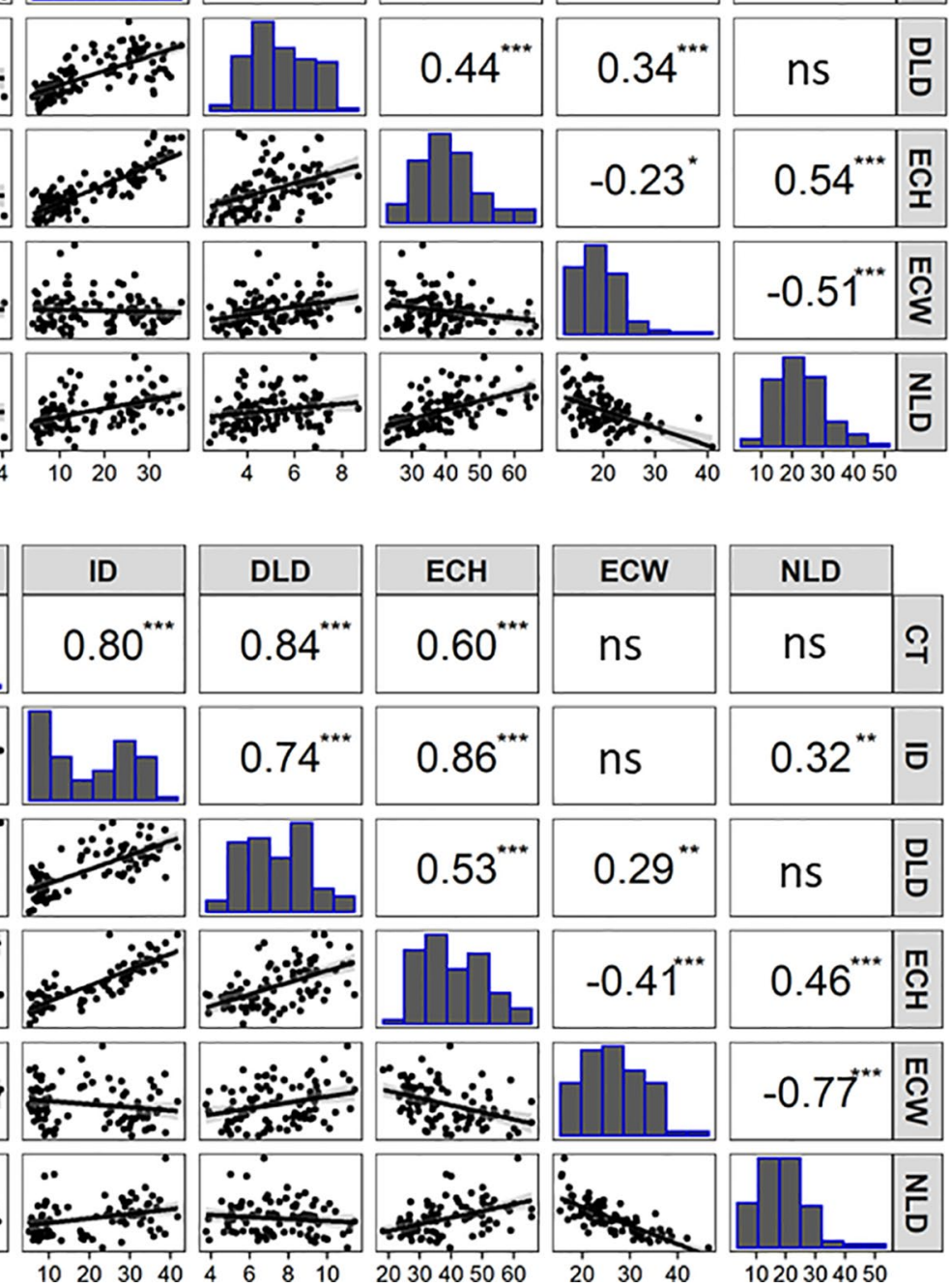

FIGURE 2 | Correlation matrix containing scatterplots, distributions, and Pearson's correlation coefficients of the cucumber fruit epidermal traits for: (A) RIL population $\left(F_{7: 8}\right)$ of Gy14 x CL9930 ( $n=110$ lines, 16 dpp) grown in the greenhouse in Fall 2016. (B) Recombinant RIL lines ( $n=87$ lines, 20 dpp) grown in the field in 2018. Cuticle thickness (CT), Intercalation depth (ID), Diameter of lipid droplets (DLD), Epidermal cell height (ECH), Epidermal cell width (ECW), and Number of lipid droplets (NLD) in $450 \mu \mathrm{m}$ linear region of epidermal cells. ( $n s$ not significant; ${ }^{\star} P<0.05 ;{ }^{* \star} P<0.01 ;{ }^{\star \star \star} P<0.001$. (Each value is the mean of three replicate fruit per RIL).

approximately 25-fold higher expression in flowers than fruit; CsaV31g030210 exhibited 50-100-fold higher expression in roots, leaves, and flowers than in fruit; and CsaV31g030360 was expressed comparably throughout the plant.

With regard to fruit development, CsSHN1 was the only gene in the QTL1 region with significantly higher expression in peels of 8 $\mathrm{dpp}$ fruit than $16 \mathrm{dpp}$ fruit (Table 2). Higher expression $(\mathrm{P}<0.05$ and 2-fold difference) of CsSHN1 at $8 \mathrm{dpp}$ than $16 \mathrm{dpp}$ also was observed in the cultivars 'Vlaspik' and 'Poinsett 76' (Figure 5A). Examination of CsSHN1 expression during cucumber fruit growth from 0-20 dpp showed a sharp window of expression (8-12 dpp) during the period of exponential fruit growth, coinciding with peak cuticle and wax deposition (Figure 5B). Consistent with observed differences in chromosome 1-associated traits of cuticle thickness, intercalation depth, and diameter of lipid droplets, expression of CsSHN1 was significantly higher in the two pickling cultigens, Gy14 and Vlaspik (Ando et al., 2015), than in CL9930. Peak expression was also somewhat delayed in CL9930, at $12 \mathrm{dpp}$ vs. 8 $\mathrm{dpp}$, corresponding with the relative timing for increase in cuticle thickness, intercalation depth, and diameter of lipid droplets. 

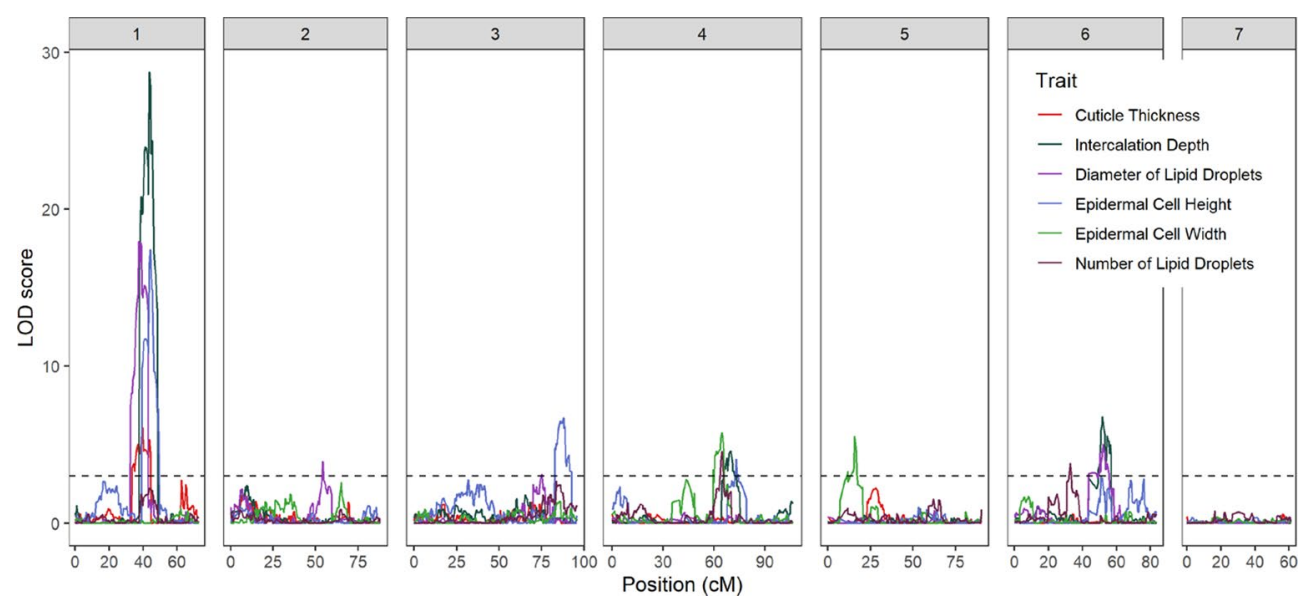

FIGURE 3 | LOD profiles of fruit epidermal QTLs detected in the RIL population across the seven chromosomes of cucumber. The dashed horizontal line is LOD threshold $=3.0$.

TABLE 1 | Summary of fruit epidermal QTLs detected: Cuticle thickness (CT), Epidermal cell height (ECH), Intercalation depth (ID), Diameter of lipid droplets (DLD), Epidermal cell width (ECW), and Number of lipid droplets (NLD).

\begin{tabular}{|c|c|c|c|c|c|c|c|c|c|c|c|}
\hline \multirow[t]{2}{*}{ Trait } & \multirow[t]{2}{*}{ Chr } & \multirow[t]{2}{*}{ QTL Loci } & \multirow{2}{*}{$\begin{array}{l}\text { Peak } \\
\text { Pos } \\
\text { (cM) }\end{array}$} & \multirow{2}{*}{$\begin{array}{l}\text { Nearest } \\
\text { Marker }\end{array}$} & \multirow{2}{*}{$\begin{array}{l}\text { LOD } \\
\text { Score }\end{array}$} & \multicolumn{4}{|c|}{ 2.0 LOD Interval } & \multirow{2}{*}{$\begin{array}{l}\text { Additive } \\
\text { Effect } \\
\text { (a) }\end{array}$} & \multirow{2}{*}{$\begin{array}{c}\text { Percent } \\
\text { Variation } \\
\text { (\%) } \mathbf{R}^{2}\end{array}$} \\
\hline & & & & & & Left Locus & Left $\mathbf{c M}$ & $\begin{array}{l}\text { Right } \\
\text { Locus }\end{array}$ & $\begin{array}{l}\text { Right } \\
\text { cM }\end{array}$ & & \\
\hline $\mathrm{CT}$ & 1 & qCT1.1 & 39.8 & SNP.120657 & 6.1 & SNP.10249 & 37.9 & SNP.9737 & 43.1 & 0.34 & 18.4 \\
\hline \multirow[t]{3}{*}{$\mathrm{ECH}$} & 1 & qECH1.1 & 44.1 & SNP.9649 & 17.4 & SNP.9649 & 43.9 & SNP.9341 & 44.9 & -6.40 & 38.1 \\
\hline & 3 & qECH3.1 & 87.8 & SNP.31473 & 6.7 & SNP.32685 & 82.8 & SNP.117081 & 89.1 & 3.60 & 11.5 \\
\hline & 4 & qECH4.1 & 73.0 & SNP.128649 & 4.1 & SNP.55545 & 71.6 & SNP.146469 & 77.3 & -2.69 & 6.7 \\
\hline \multirow[t]{3}{*}{ ID } & 1 & qID1.1 & 43.6 & SNP.9649 & 28.7 & SNP.9649 & 43.5 & SNP.9649 & 44.1 & -7.50 & 58.3 \\
\hline & 4 & qID4.1 & 69.8 & SNP.55081 & 4.6 & SNP.140945 & 67.9 & SNP.128649 & 72.6 & -2.07 & 4.5 \\
\hline & 6 & qID6.1 & 51.8 & SNP.85409 & 6.8 & SNP.84997 & 50.7 & SNP.86689 & 53.1 & -2.66 & 6.9 \\
\hline \multirow[t]{3}{*}{ DLD } & 1 & qDLB1.1 & 37.4 & SNP.9941 & 18.0 & SNP.9941 & 37.0 & SNP.10249 & 37.9 & -0.94 & 42.5 \\
\hline & 2 & $q D L B 2.1$ & 54.2 & SNP.143745 & 3.9 & SNP.23461 & 52.8 & SNP.131577 & 56.5 & 0.38 & 6.9 \\
\hline & 6 & $q D L B 6.1$ & 52.2 & SNP.175561 & 5.0 & SNP.84997 & 50.8 & SNP.128265 & 53.6 & -0.44 & 8.8 \\
\hline \multirow[t]{2}{*}{ ECW } & 4 & qECW4.1 & 64.6 & SNP.52733 & 5.8 & SNP.55017 & 61.8 & SNP.53205 & 65.7 & 2.23 & 14.8 \\
\hline & 5 & qECW5.1 & 15.7 & SNP.76545 & 5.5 & SNP.134473 & 15.2 & SNP.77021 & 17.0 & 2.09 & 14.5 \\
\hline \multirow[t]{2}{*}{ NLD } & 4 & qNLB4.1 & 64.6 & SNP.52733 & 4.6 & SNP.122297 & 63.9 & SNP.53205 & 65.5 & -2.96 & 12.6 \\
\hline & 6 & qNLB6.1 & 32.8 & SNP.151005 & 3.8 & SNP.90761 & 30.8 & SNP.89757 & 35.6 & -3.53 & 10.3 \\
\hline
\end{tabular}

Negative additive effect values (a) indicate that the allele is derived from parent Gy14. Positive additive effect values (a) indicate that the allele is derived from parent CL9930.

The predicted length of CsSHN1 is $957 \mathrm{bp}$; transcript data (http://cucurbitgenomics.org/) support a single intron, consistent with other SHINE genes (Borisjuk et al., 2014). Comparison of the coding region plus $2 \mathrm{~Kb}$ upstream between Gy14 and CL9930 identified a SNP, within exon 2. The Vlaspik and Poinsett 76 sequences also shared the Gy14 sequence. A KASP marker was designed for the SNP at position 16961026 on chromosome 1 (CL9930 v. 3). The allele present (CL9930 vs. Gy14) at this position in the RILs completely co-segregated with phenotype. Marked allele effects were observed for the four fruit epidermal traits (Figure 5C).

The SNP at this position ('C' in Gy14 vs. 'G' in CL9930) results in a predicted amino acid change, from proline in Gy14 to arginine in CL9930, within a highly conserved region of the protein [domain CMV-1 as per Nakano et al. (2006)]. All of the other cucurbits for which there are draft genomes [Citrullus lanatus, Cucumis melo, Cucurbita maxima, Cucurbita moschata, Cucurbita pepo, Cucurbita argyrosperma, Lagenaria siceraria (http://cucurbitgenomics.org/)] like Gy14, contain proline in this position (Supplementary Table 5). In addition, more than 30 divergent plant species with homologs identified by BLAST, also contain a proline residue at this position (Supplementary Table 7). Within cucumber germplasm, however, the CL9930 variant is quite common. Of 140 re-sequenced accessions with $\geq 10$ reads at this position, 44 exhibited the CL9930 allele; another nine are heterozygous at this position (Supplementary Table 4).

\section{DISCUSSION}

\section{Variation in Epidermal Properties of Cucumber Fruit During Development}

Cucumber fruit sampled at incremental ages from anthesis through maturity were characterized for developmental changes and natural variation for epidermal traits, including epidermal 


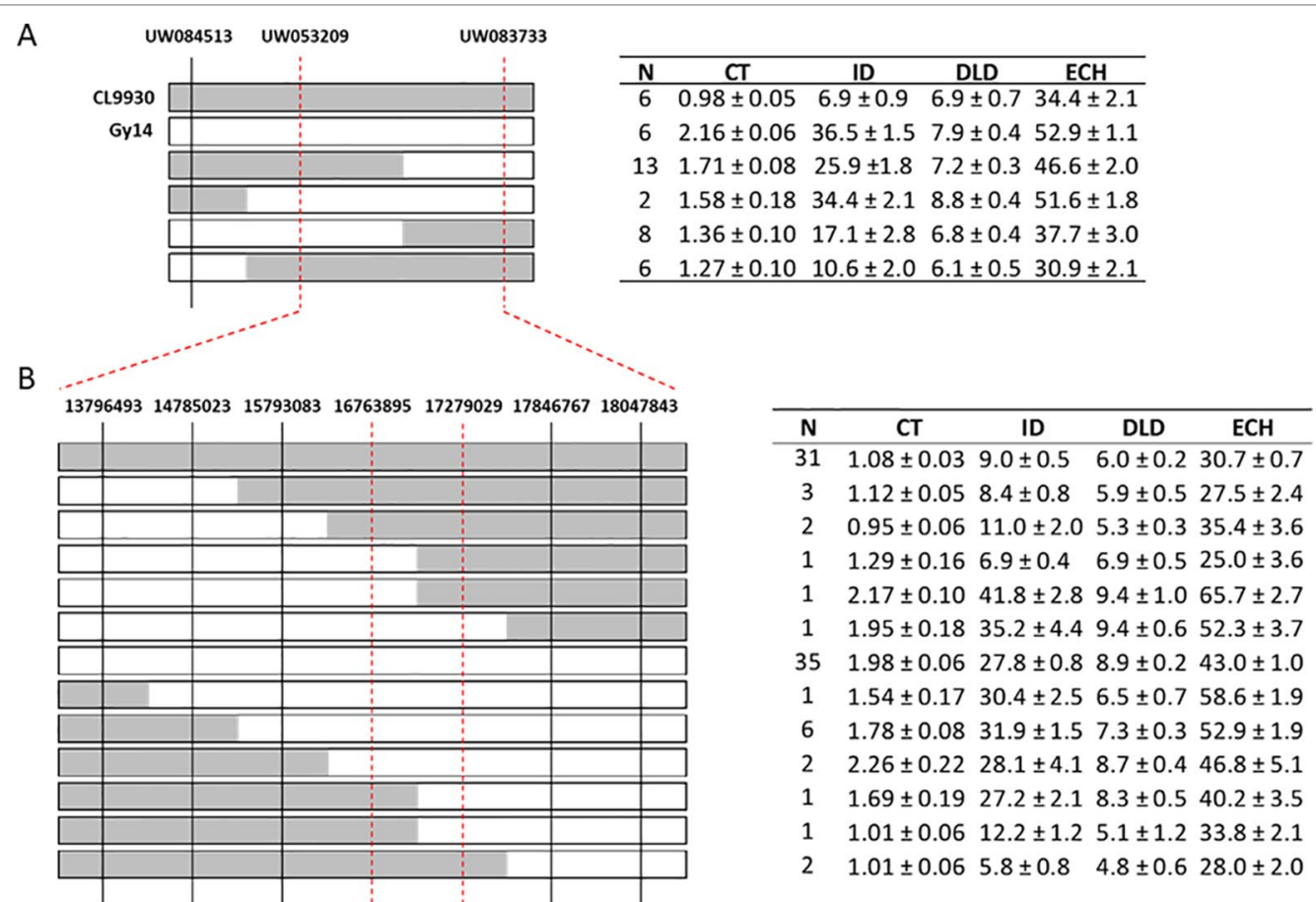

FIGURE 4 | Fine mapping of chromosome 1. (A) Genotyping of recombinant plants from an expanded $F_{7: 8}$ RIL population using SSR markers ( $n=375$ ); 87 lines were selected of which 29 were recombinant in the region of interest on chromosome 1. Parental lines, CL9930 and Gy14, are included as reference. CL9930 alleles are denoted by grey, Gy14 by white. (B) Additional genotyping of expanded RIL population using KASPTM markers in narrowed region of chromosome 1. Phenotypes of RILs sharing a given recombinant type are indicated in the adjacent tables. Measurements of traits are as for Figure $\mathbf{3}$. Trait values ( $\mu$ m) are the mean \pm S.E. of all RILs sharing a given recombinant type (three biological replicates/RIL).

cell shape, cuticle thickness, cuticular intercalations between epidermal cells, and the number and size of lipid droplets. Fruits from both Gy14 and CL9930 followed a characteristic sigmoidal pattern of growth, consistent with previous studies (Colle et al., 2017). The associated epidermal fruit traits also exhibited this pattern, with the greatest increase occurring at 4-12 days post pollination, coinciding with the period of exponential growth. The period of peak deposition of cuticle and wax during the period of maximal cucumber fruit growth is consistent with other systems where cuticle and wax deposition is developmentally programmed, often ceasing during early fruit development (Hen-Avivi et al., 2014; Martin and Rose, 2014; Lara et al., 2015; Trivedi et al., 2019). Beginning with the commencement of exponential growth and continuing throughout development, Gy14 had consistently larger values for cuticle thickness, cuticular intercalation, and number and size of lipid droplets. By $16 \mathrm{dpp}$, fruit size and differences in epidermal traits had largely plateaued; therefore, 16-20 dpp became the benchmark age for further epidermal work.

A striking observation was the presence of numerous large lipid droplets, typically $4-10 \mu \mathrm{m}$, in the epidermal cells. In plants, lipid droplets are thought to be formed in the endoplasmic reticulum and surrounded by a monolayer of phospholipids and structural membrane proteins (Chapman et al., 2012; Huang, 2018; Shimada et al., 2018). Lipid droplets in plants can vary quite widely in size, ranging from $<1$ to $\sim 20 \mu \mathrm{m}$, depending on species and organ or tissue; larger ones are more frequently found in oil rich fruit tissues (Goold et al., 2015). Much of what is known about the roles of lipid droplets comes from research involving seeds and leaves, but studies of fruits of avocado, olive, and oil palm suggest that the lipid droplets likely have varying functions for different tissue types (Pyc et al., 2017). Originally, lipid droplets were thought to have functions restricted to lipid storage, but recent findings have suggested that lipid droplets can be involved in more complex processes, such as lipid signaling and disease resistance (Chapman et al., 2012; Pyc et al., 2017). Lipid droplets also can sequester lipidsoluble compounds such as terpenoids that may contribute to protection against fungal or oomycete pathogens (e.g., Shimada et al., 2018; Sadre et al., 2019). Whether the cucumber fruit lipid droplets function in other capacities such as defense remains to be investigated.

\section{Mapping of, and Relationships Among, Epidermal Fruit Traits}

Phenotypic analysis of the Gy14 × CL9930 RIL populations was performed to ascertain genetic factors underlying the variation in epidermal traits. QTL for the six epidermal traits were detected on six of the seven cucumber chromosomes. Given the large LOD profiles and high correlation of traits mapping to chromosome 1 , SSR markers designed to cover the peak QTL region on chromosomes 1 were used to screen an expanded Gy14 $\times$ CL9930 $\mathrm{F}_{7: 8}$ RIL population for recombinants in this region. 
TABLE 2 | Annotated genes within fine-mapped region of QTL on cucumber chromosome 1 (16.764-17.279 Mb). Chromosomal positions and annotations are derived from Chinese Long v. 3.

\begin{tabular}{|c|c|c|c|c|c|c|c|}
\hline \multirow[t]{3}{*}{ Gene ID } & \multirow{2}{*}{\multicolumn{2}{|c|}{$\begin{array}{c}\begin{array}{c}\text { Fruit expression } \\
\text { (2 wks post-pollination) }\end{array} \\
\text { CL9930 }\end{array}$}} & \multicolumn{2}{|c|}{ Peel expression } & \multirow[t]{3}{*}{ Description } & \multirow{2}{*}{\multicolumn{2}{|c|}{ Arabidopsis Homolog }} \\
\hline & & & \multicolumn{2}{|c|}{ Gy14b } & & & \\
\hline & Flesh & Peel & $8 \mathrm{dpp}$ & 16 dpp & & & e Value \\
\hline CsaV31g030010 & 16 & 12 & 17.5 & 91.5 & nitrate transporter & At2g26690 & $2.5 e-245$ \\
\hline CsaV31g030020 & 12.2 & 9.7 & 11.2 & 15.1 & similarity to DNA helicase & At1g65810 & $7.3 e-23$ \\
\hline CsaV31g030030 & 0 & 0 & 0 & 0 & Zn binding dehydrogenase family & At5g16990 & $3.5 e-23$ \\
\hline CsaV31g030090 & 13.9 & 21.7 & 57.7 & 42.9 & heme oxygenase & At2g26670 & $9.2 \mathrm{e}-111$ \\
\hline CsaV31g030110 & 8.5 & 2.2 & 2.4 & 4.7 & metal transporter & At5g53550 & $3.2 \mathrm{e}-286$ \\
\hline CsaV31g030120 & 0 & 0 & 2.6 & 0.9 & peroxidase superfamily & At2g41480 & 8.4 e-95 \\
\hline CsaV31g030140 & 0 & 0 & 0 & 0 & TIR-NBS-LRR protein & At5g17680 & $7.2 \mathrm{e}-157$ \\
\hline CsaV31g030160 & 14.1 & 19.8 & 0 & 0 & unknown & no match & \\
\hline CsaV31g030170 & 0 & 0 & 0 & 0 & peroxidase superfamily & At2g41480 & $4.3 e-117$ \\
\hline CsaV31g030190 & 7.8 & 9.2 & 9.1 & 5.5 & SPX domain containing & At2g26660 & $1.0 e-100$ \\
\hline CsaV31g030200 & 1.4 & 115.8 & 343.5 & 13.3 & SHINE1MIN1 & At1g15360 & $1.2 \mathrm{e}-57$ \\
\hline CsaV31g030210 & 8.3 & 18.5 & 7.2 & 11 & unknown & At1g52565 & $2.1 \mathrm{e}-12$ \\
\hline CsaV31g030220 & 124.2 & 99.5 & 86.7 & 99.8 & 50 S ribosomal L36 & At5g20180 & $2.3 e-30$ \\
\hline CsaV31g030230 & 28.4 & 21.7 & 40.8 & 30.7 & DUF 4050 family protein & At5g25360 & $2.5 e-58$ \\
\hline CsaV31g030250 & 0.1 & 0.5 & 0.5 & 0.1 & EIN3 binding F-box protein-like & At5g25350 & $1.8 e-84$ \\
\hline CsaV31g030300 & 175.0 & 128.5 & 100.7 & 106.2 & tetratricopeptide repeat protein & At5g20190 & $2.1 e-25$ \\
\hline CsaV31g030310 & 0.9 & 0.7 & 0.3 & 6.4 & nucleotide transporter like & At1g02630 & $1.0 \mathrm{e}-133$ \\
\hline CsaV31g030330 & 22.9 & 28.2 & 14.5 & 23.7 & nuclear pore complex protein & At5g20200 & $7.8 e-82$ \\
\hline CsaV31g030340 & 0 & 0 & 0 & 0 & axial regulator YABBY-like & At2g26580 & $2.4 \mathrm{e}-62$ \\
\hline CsaV31g030350 & 8.7 & 8.1 & 13.8 & 7.6 & methyl CpG binding domain & At3g15790 & $3.4 \mathrm{e}-29$ \\
\hline CsaV31g030360 & 6.8 & 13.1 & 15.5 & 16.3 & glucan endo-1-3 beta glucosidase & At2g26600 & $3.4 e-29$ \\
\hline CsaV31g030390 & 0.2 & 0 & 2.1 & 11.1 & blue copper protein & At5g20230 & $4.7 \mathrm{e}-16$ \\
\hline CsaV31g030400 & 11.4 & 11.3 & 12.9 & 14.9 & transducin family protein & At2g26610 & 0 \\
\hline CsaV31g030420 & 12.4 & 13.9 & 14.6 & 12.4 & acetylgluosaminyl transferase family & At2g13290 & $6.8 e-143$ \\
\hline CsaV31g030440 & 0 & 0 & 0 & 0 & pentatricopeptide repeat containing & At4g02750 & $1.2 \mathrm{e}-15$ \\
\hline
\end{tabular}

Predicted genes with no homology match and no expression in fruit were not included.

aExpression data from Wei et al., 2016. Accessed via http://cucurbitgenomics.org/, Gene expression project PRJNA 312872.

${ }^{b}$ Expression data from Mansfeld et al., 2017. Available via http://cucurbitgenomics.org/, Gene expression project PRJNA 345040.

Bold indicates higher expression in peel than flesh and higher expression at $8 \mathrm{dpp}$ than $16 \mathrm{dpp}$.

Phenotyping of identified recombinants were narrowed to a region of $\sim 3 \mathrm{Mb}$ on chromosome 1 . Fine mapping of the region of interest on chromosome 1 using $\mathrm{KASP}^{\mathrm{TM}}$ markers narrowed the region to an area of $\sim 0.5 \mathrm{Mb}$. The very strong correlation between phenotype traits in the field and greenhouse indicate that, despite greatly differing environments, the measured traits on chromosome 1 are predominantly affected by genotype and developmental stage, rather than environment.

Strong, positive correlations were observed for cuticle thickness, intercalation depth, epidermal cell height, and diameter of lipid droplets along with a strong QTL on chromosome 1 . While there was variation among RILS for relative intercalation depth (i.e., not all long cells had deep intercalations) the very strong correlation between epidermal cell height and intercalation depth argues that cell structure may be an important factor influencing cuticular intercalations in cucumber fruit. Diversity in intercalation patterns has been observed in a variety of species as illustrated by a 'grocery store survey' of numerous fruit types (Martin and Rose, 2014). The origin of such material remains unclear, possibly due to detachment from the epidermal cuticle and downward movement, or direct deposition to the anticlinal cell wall regions. It is also not clear whether diversity in intercalation patterns result from active regulation or from mechanical constraints of the cell structure (Martin and Rose, 2014). Consistent with a possible role of cell structure, in addition to the major QTL for intercalation depth and epidermal cell height on cucumber chromosome 1, intercalation depth also appears to share a QTL with epidermal cell height and cell width on chromosome 4.

Epidermal cell height and width showed a modest, negative association, and RIL phenotyping displayed a wide range of cell shapes beyond that of the flat and palisade orientations characteristic of CL9930 and Gy14, respectively. This variation is likely due to multiple factors controlling epidermal cell shape, including the $P e$ gene on chromosome 5. Pe has been localized to a $0.23 \mathrm{Mb}$ region and exhibits tight, but not unbreakable, linkage to several fruit surface related traits such as dull $(D)$, uniform fruit color $(u)$ and tuberculate $(T u)$, suggesting a cluster of genes modulating cucumber exocarp characteristics (Yang et al., 2014a, Yang et al., 2014b, Yang et al., 2014c; Chen et al., 2016; Zhang et al., 2017; Yang et al., 2019). The QTL for epidermal cell width identified on chromosome 5 in this study is consistent with the location of $\mathrm{Pe}$. Interestingly, the number of lipid droplets was not significantly related to size of lipid droplets suggesting that multiple factors regulate lipid droplet formation. Consistent with this observation, QTL for number of lipid body number and size were present on different chromosomes, 4 and 6 for number, and 1, 2 and 6, for size. 

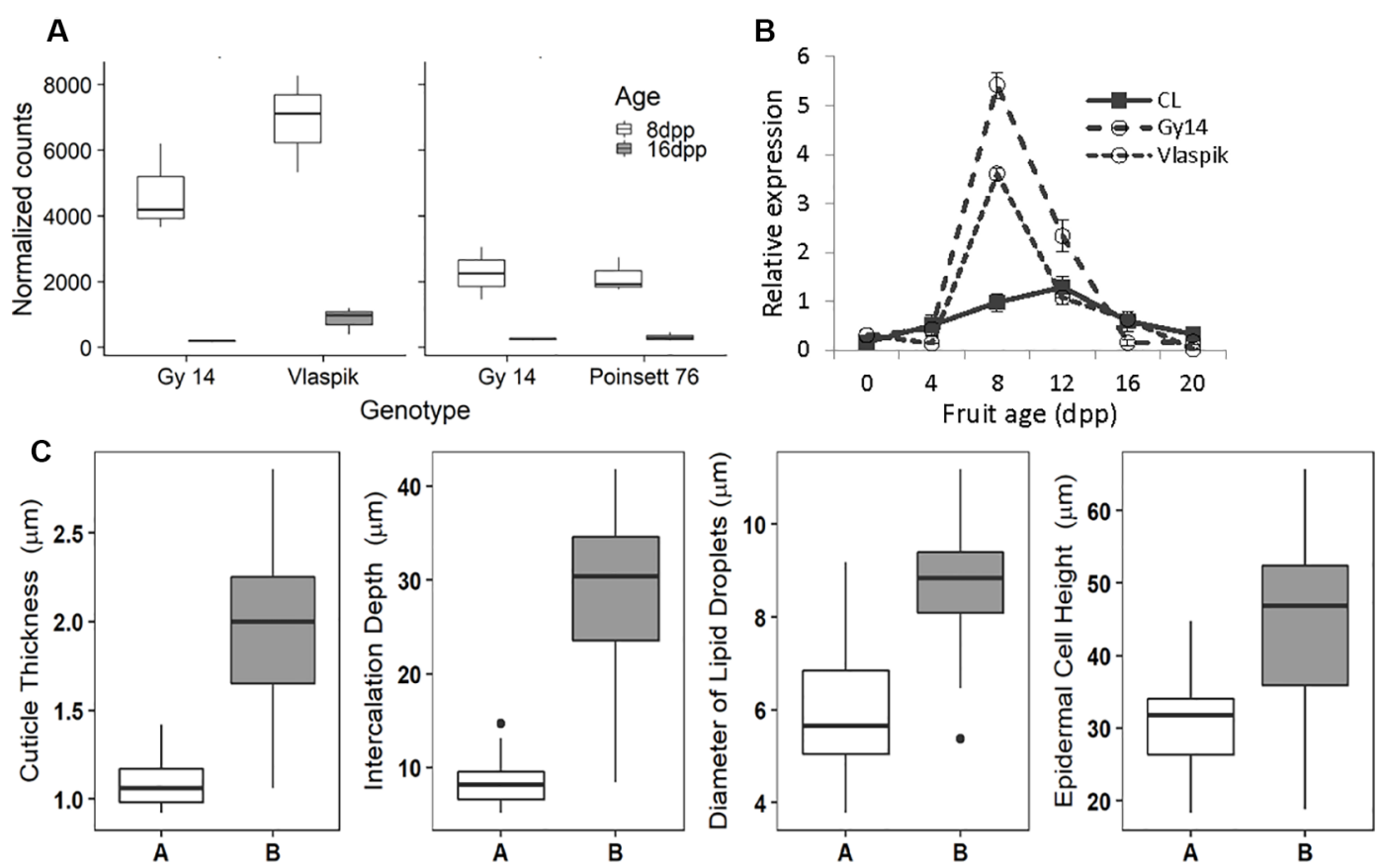

FIGURE 5 | Transcriptional analysis and allele effect of the CsSHINE1 (CsaV3_1g030200). (A) Comparison of expression of CsSHN1 in fruit peel from cucumber fruit at 8 and 16 days post pollination (dpp). (B) Expression of CsSHN1 in CL9930 and two pickling cultivars, Gy14 and Vlaspik, during cucumber fruit development, from anthesis $(0 \mathrm{dpp})$ to $20 \mathrm{dpp}$. Each value is the mean of 3 biological replicates with 3 technical replicates/biological replicate \pm S.E. (C) Allele effect of CsSHN1 SNP at position 16961026 (in Chinese Long V3) for cuticle thickness, intercalation depth, diameter of lipid droplets and epidermal cell height as assessed from the expanded RIL population. 'A' refers to RILs with CL9930 allele $(n=25)$ and 'B' to RILs with the Gy14 allele $(n=35)$.

\section{CsSHN1 is a Candidate Gene Influencing Cucumber Fruit Surface Properties}

Mapping results and SSR and KASP marker assay refined the major QTL on chromosome 1 to a region containing 25 annotated genes. Expression profiles of these genes showing peak transcription coinciding with period of rapid fruit growth and deposition of cuticle, strongly preferential expression in fruit exocarp, and known function of SHINE transcription factors as regulators of cuticle and wax deposition (Yeats and Rose, 2013; Hen-Avivi et al., 2014; Trivedi et al., 2019), collectively implicate CsSHN1 (Csalg340430) as the primary candidate gene underlying the chromosome 1 QTL. SHN (SHINE) or WIN (WAX INHIBITOR) genes are members of the apetala2/ethylene-responsive element biniding protein (ap2/ere bp) transcription factor family originally named in Arabidopsis for their role in leaf appearance and the regulation of cuticle biosynthesis (Aharoni et al., 2004; Broun et al., 2004). SHN genes are primarily expressed in epidermal tissue in locations and periods of rapid growth, allowing for coverage and protection of the developing organ (Hen-Avivi et al., 2014; Trivedi et al., 2019). Expression of CsSHN1 in cucumber fruit was consistent with this pattern, and mirrors the tissue specific and developmental regulation observed for SlSHN3 in tomato fruit (Shi et al., 2013). Similar to SlSHN3, CsSHN1 is nearly exclusively expressed in exocarp of immature fruit relative to other organs, tissues and ages.
Several studies have demonstrated that overexpression of SHN homologs increases wax deposition and cuticle thickness by modulating expression of cutin and wax biosynthesis genes, either directly or indirectly; conversely, down-regulation results in reduced cuticle and waxes (e.g., Aharoni et al., 2004; Broun et al., 2004; Kannangara et al., 2007; Shi et al., 2013). Variants for cuticle and wax deposition have primarily identified by mutant screens; however, more recent genomic, transcriptomic and metabolomic approaches have enabled the identification of natural variants (Cohen et al., 2017). While the majority of cuticle and wax variants identified to date include biosynthetic enzymes and lipid transporters [e.g., fatty acid omega hydroxylase (CYP861A, CYP86B1), BAHD acetyltransferase, beta-ketoacyl-CoA synthase, triterpene synthases, GDSL lipase] (Cohen et al., 2017), it has been suggested that regulatory genes are the most likely targets to achieve fine modulation (Petit et al., 2017). Naturally occurring variation for the naked caryopsis phenotype in barley, a trait causing loss of a sticky lipid substance secreted by the epidermis, was found to arise from mutation in a SHN1 allele in barley (Taketa et al., 2008; Taketa et al., 2012) and genomic studies in apple have suggested that variations in the apple homolog of SHN1 (MdSHN3), influence cuticle formation and russeting disorder in apple fruit (Lashbrooke et al., 2015b).

CsSHN1, like other SHINE and AP2/EREBP proteins includes the highly conserved ERF domain in the amino terminal portion of the protein. SHINE proteins are assigned to Group V of the 
AP2/EREBP family (Nakano et al., 2006; Borisjuk et al., 2014). Group $\mathrm{V}$ includes a single intron and two conserved domains, CMV-1 and CMV-2, toward the middle and C-terminal portion of the protein, respectively. These features also occur in CsSHN1. The substitution of arginine for proline in CL9930 vs. Gy14 occurs within the conserved CMV-1 domain [also referred to as middle motif 'mm' (Aharoni et al., 2004)]. Mutation of a valine to aspartic acid mutation in this motif was shown to cause the naked caryopsis phenotype in barley, indicating functional significance of this domain (Taketa et al., 2008; Taketa et al., 2012). It remains to be determined whether the observed mutation in CsSHN1 influences activity of the CsSHN1 transcription factor. The phenotypic differences observed between Gy14 and CL9930 may reflect protein activity and/or expression levels, as CL9930 also had reduced expression relative to Gy14. We did not observe sequence differences within the promoter $(2 \mathrm{~kb}$ upstream of the coding region) or intron, suggesting that effects on transcript levels may result from other more distant elements or from relative RNA stability.

Despite conservation of the proline at this position among more than thirty species examined, including both dicots and monocots, the substitution was quite common among cucumber accessions (present in approximately a third of the re-sequenced lines). Cucumber is thought to have been first domesticated in South Asia and then subsequently moved both east toward China and west toward Europe, forming three major clades (Lv et al., 2012; Qi et al., 2013; Wang et al., 2018). Although there are exceptions, the CL9930 allele is predominantly found (70\%) in East Asian accessions where it is widely present in cultivated East Asia cucumbers, but not landraces. This may suggest that this gene is under selection in the making of East Asia cucumbers (long, thin skin). The more frequent Gy14 allele is in present in many cultivars, landraces, semi-wild and wild cucumbers. Interestingly, though, it appears that the CL9930 allele also may be present at a relatively low frequency in the wild C. sativus var. hardwickii, as one of the 12 re-sequenced hardwickii accessions possessed this variant. This may reflect possible occurrence prior to domestication. Alternatively, as gene flow between cultivated cucumber and hardwickii populations occurs in natural populations (Bisht et al., 2004; Yang et al., 2012), this variation may have originated after domestication.

Several lines of evidence additionally suggest interplay between cuticle deposition and epidermal cell differentiation and development (e.g., Javelle et al., 2010; Nadakuduti et al., 2012; Yeats and Rose, 2013; Hen-Avivi et al., 2014; FernandezMoreno et al., 2017). This also has been observed for members of the SHINE family. For example, overexpression of Arabidopsis SHN1 altered epidermal cell structure, including formation of elongated cells and reduced stomatal density and trichome number (Aharoni et al., 2004) and tomato SlSHN3 influences cell shape, either directly, or indirectly by influencing expression of other cell downstream patterning genes such as SIMIXTA (Shi et al., 2013; Lashbrooke et al., 2015a). Down regulation of SlSHN3 results in reduced cuticle deposition and flattened fruit epidermal cells. The connection between SHINE-family member genes and cell shape may also contribute to the observed QTL for cucumber epidermal cell height at this location.

\section{CONCLUSIONS}

Cucumber fruit epidermis exhibits dynamic developmental changes during fruit growth including changes in cell size and shape, deposition of cuticle, and appearance of lipid droplets. There is also natural variation for these traits as manifest in differing cucumber market classes, and observed for the Chinese fresh market cucumber, CL9930, relative to the American pickling cucumber, Gy14. Genetic analyses indicated several QTL, including a major QTL on chromosome 1, QTL ECT1.1, influencing cuticle thickness and depth of intercalation between epidermal cells, diameter of lipid droplets and epidermal cell height. Additional QTL of lesser impact were present on chromosomes 3, 4, 5 and 6. Fine mapping of the four traits associated with QTL ECT1.1 narrowed the region to $0.5 \mathrm{Mb}$. Transcriptomic analysis based on tissue-specific and developmentally-regulated expression of fruit epidermal traits of genes in this region along with and observed allelic effects, identified a primary candidate gene-a homolog of SHINE1, which in other systems has been shown to influence both cuticle deposition and epidermal cell shape. The CsSHN1 sequence in CL9930 includes a single base difference causing an amino acid change (proline to arginine) in the highly conserved CMV-1 domain when compared to that in Gy14. This single base change, which occurred frequently in East Asian cucumber accessions may contribute to natural variation for cucumber epidermal properties. As epidermal properties, including wax deposition, influence both consumer preferences and longevity in the market chain, allelic variation in CsSHN1 may provide a valuable target for breeders developing varieties to meet desired fruit quality characteristics, such as fruit with shinier appearance (reduced wax) or extended shelf life due to reduced water loss (increased wax).

\section{DATA AVAILABILITY STATEMENT}

The datasets generated for this study can be found in the NCBI Sequence Read Archive (SRA) under project accession number PRJNA558838.

\section{AUTHORS CONTRIBUTIONS}

SR-C, RG and CB conceived of the project. SR-C and RG wrote the paper. SR-C performed the developmental analyses, phenotyping of the RILs, QTL mapping, KASP analyses and fine mapping. MC performed the developmental analysis of CsSHN1 expression. BM performed the RNA-Seq experiment, differential gene expression analysis and SNP calling of parental lines. YWa and YWe performed the SSR analysis and identification of recombinants. $\mathrm{LG}$ and $\mathrm{ZF}$ analyzed the re-sequenced cucumber accessions for alleles of CsSHN1.

\section{FUNDING}

This research was in part supported by BARD, The United StatesIsrael Binational Agricultural Research and Development Fund, Research Grant Award No. US-5009-17; the National Institute of Food and Agriculture (NIFA), U.S. Department of Agriculture, 
Award No. 2015-51181-24285, and by USDA NIFA Hatch project number MICL02349 to RG and MICL02552 to CB.

\section{ACKNOWLEDGEMENTS}

We would like to thank Drs. Courtney Hollender, Linda Hanson and Frank Telewski (MSU) for the use of their microscopy and microtome equipment and Dr. Andrew Wiersma (MSU) for his assistance with the establishing of the KASP genotyping assay.

\section{REFERENCES}

Adato, A., Mandel, T., Mintz-Oron, S., Venger, I., Levy, D., Yativ, M., et al. (2009). Fruit-surface flavonoid accumulation in tomato is controlled by a SlMYB12regulated transcriptional network. PloS Genet. 5, e1000777. doi: 10.1371/ journal.pgen.1000777

Aharoni, A., Dixit, S., Jetter, R., Thoenes, E., van Arkel, G., and Pereiraa, A. (2004). The SHINE clade of AP2 domain transcription factors activates wax biosynthesis, alters cuticle properties, and confers drought tolerance when overexpressed in Arabidopsis. Plant Cell 16, 2463-2480. doi: 10.1105/ tpc.104.022897

Ando, K., Carr, K. M., and Grumet, R. (2012). Transcriptome analysis of early cucumber fruit growth identifies distinct gene modules associated with phases of development. BMC Genomics 13, 518. doi: 10.1186/1471-2164-13-518

Ando, K., Carr, K. M., Colle, M., Mansfeld, B. N., and Grumet, R. (2015). Exocarp properties and transcriptomic analysis of cucumber (Cucumis sativus) fruit expressing resistance to Phytophthora capsici. PloS One 10, e0142133. doi: 10.1371/journal.pone.0142133

Ando, K., Grumet, R. 2010. Transcriptional profiling of rapidly growing cucumber fruit by 454-pyrosequencing analysis. J. Amer. Soc. Hortic. Sci. 135, 291-302. doi: 10.21273/JASHS.135.4.291

Benjamini, Y., and Hochberg, Y. (1995). Controlling the false discovery rate: A practical and powerful approach to multiple testing. J. R. Stat. Soc B. 57, 289300. doi: 10.1111/j.2517-6161.1995.tb02031.x

Bisht, I. S., Bhat, K. V., Tanwar, S. P. S., Bhandari, D. C., Joshi, K., and Sharma, A. K. (2004). Distribution and genetic diversity of Cucumis sativus var. hardwickii (Royle) Alef in India. J. Hortic. Sci. Biotech. 79, 783-791. doi: 10.1080/14620316.2004.11511843

Bolger, A. M., Lohse, M., and Usadel, B. (2014). Trimmomatic: a flexible trimmer for Illumina sequence data. Bioinformatics 30, 2114-2120. doi: 10.1093/ bioinformatics/btu170

Borisjuk, N., Hrmova, M., and Lopato, S. (2014). Transcriptional regulation of cuticle biosynthesis. Biotechnol. Adv. 32, 526-540. doi: 10.1016/j.biotechadv. 2014.01.005

Broun, P., Poindexter, P., Osborne, E., Jiang, C.-Z., and Riechmann, J. L. (2004). WIN1, a transcriptional activator of epidermal wax accumulation in Arabidopsis. Proc. Natl. Acad. Sci. U.S.A. 101, 4706-4711. doi: 10.1073/ pnas.0305574101

Buda, G. J., Isaacson, T., Matas, A. J., Paolillo, D. J., and Rose, J. K. C. (2009). Threedimensional imaging of plant cuticle architecture using confocal scanning laser microscopy. Plant J. 60, 378-385. doi: 10.1111/j.1365-313X.2009.03960.x

Chapman, K. D., Dyer, J. M., and Mullen, R. T. (2012). Biogenesis and functions of lipid droplets in plants. J. Lipid Res. 53, 215-226. doi: 10.1194/ jlr.R021436

Chen, C. H., Yin, S., Liu, X. W., Liu, B., Yang, S., Xue, S. D., et al. (2016). The WD-repeat protein CsTTG1 regulates fruit wart formation through interaction with the Homeodomain-Leucine zipper I protein Mict. Plant Physiol. 171, 1156-1168. doi: 10.1104/pp.16.00112

Cohen, H., Szymanski, J., and Aharoni, A. (2017). Assimilation of 'omics' strategies to study the cuticle layer and suberin lamellae in plants. J. Exp. Bot. 68, 53895400. doi: 10.1093/jxb/erx348

Colle, M., Weng, Y., Kang, Y., Ophir, R., Sherman, A., and Grumet, R. (2017). Variation in cucumber (Cucumis sativus L.) fruit size and shape results from
We also thank the Michigan State University Research Technology Support Facility Genomics Core for genomic and RNA-Seq library preparation and sequencing.

\section{SUPPLEMENTARY MATERIAL}

The Supplementary Material for this article can be found online at: https://www.frontiersin.org/articles/10.3389/fpls.2019.01536/ full\#supplementary-material

multiple components acting pre-anthesis and post-pollination. Planta 246, 641-658. doi: 10.1007/s00425-017-2721-9

Colle, M. (2015). Cucumber (Cucumis sativus L.) fruit development: factors influencing fruit size, shape and resistance to Phytophthora capsisi. East Lansing MI: PhD Dissertation, Michigan State University.

DePristo, M. A., Banks, E., Poplin, R., Garimella, K. V., Maguire, J. R., Hartl, C., et al. (2011). A framework for variation discovery and genotyping using nextgeneration DNA sequencing data. Nat. Genet. 43, 491-498. doi: 10.1038/ng.806

Fernandez-Moreno, J. P., Levy-Samoha, D., Malitsky, S., Monforte, A. J., Orzaiz, D., Aharoni, A., et al. (2017). Uncovering tomato quantitative trait loci and candidate genes for fruit cuticular lipid composition using the Solanum pennellii introgression line population. J. Exp. Bot. 68, 2703-2716. doi: 10.1093/ jxb/erx134

Fu, F. Q., Mao, W. H., Shi, K., Zhou, Y. H., and Yu, J. Q. (2010). Spatio-temporal changes in cell division, endoreduplication and expression of cell cycle-related genes in pollinated and plant growth substances-treated ovaries of cucumber. Plant Biol. 12, 98-107. doi: 10.1111/j.1438-8677.2009.00203

Gao, M. L., Hu, L. L., Li, Y. H., and Weng, Y. (2016). The chlorophyll-deficient golden leaf mutation in cucumber is due to a single nucleotide substitution in CsChlI for magnesium chelatase I subunit. Theor. Appl. Genet. 129, 1961-1973. doi: 10.1007/s00122-016-2752-9

Gimenez, E., Dominguez, E., Pineda, B., Heredia, A., Moreno, V., Lozano, R., et al. (2015). Transcriptional activity of the MADS box ARLEQUIN/TOMATO AGAMOUS-LIKE1 gene is required for cuticle development of tomato fruit. Plant Physiol. 168, 1036-1048. doi: 10.1104/pp.15.00469

Goold, H., Beisson, F., Peltier, G., and Li-Beisson, Y. (2015). Microalgal lipid droplets: composition, diversity, biogenesis and functions. Plant Cell Rep. 34 545-555. doi: 10.1007/s00299-014-1711-7

Hen-Avivi, S., Lashbrooke, J., Costa, F., and Aharoni, A. (2014). Scratching the surface: genetic regulation of cuticle assembly in fleshy fruit. J. Exp. Bot. 65, 4653-4664. doi: 10.1093/jxb/eru225

Huang, S., Li, R., Zhang, Z., Li, L., Gu, X., Fan, W., et al. (2009). The genome of the cucumber, Cucumis sativus L. Nat. Genet. 41, 1275-1281. doi: 10.1038/ng.475

Huang, A. H. C. (2018). Plant lipid droplets and their associated proteins: potential for rapid advances. Plant Physiol. 176, 1894-1918. doi: doi.org/10.1104/pp.17.01677

Ingram, G., and Nawrath, C. (2017). The roles of the cuticle in plant development: organ adhesions and beyond. J. Exp. Bot. 68, 5307-5321. doi: 10.1093/jxb/ erx313

Isaacson, T., Kosma, D. K., Matas, A. J., Buda, G. J., He, Y., Yu, B., et al. (2009). Cutin deficiency in the tomato fruit cuticle consistently affects resistance to microbial infection and biomechanical properties, but not transpirational water loss. Plant J. 60, 363-377. doi: 10.1111/j.1365-313X.2009.03969.x

Javelle, M., Vernoud, V., Rogowsky, P. M., and Ingram, G. C. (2010). Epidermis: the formation and functions of a fundamental plant tissue. New Phytol. 189, 17-39. doi: 10.1111/j.1469-8137.2010.03514

Kannangara, R., Branigan, C., Liu, Y., Penfield, T., Rao, V., Mouille, G., et al. (2007). The transcription factor WIN1/SHN1 regulates cutin biosynthesis in Arabidopsis thaliana. Plant Cell. 19, 1278-1294. doi: 10.1105/tpc.106.047076

Kunst, L., and Samuels, A. L. (2003). Biosynthesis and secretion of plant cuticular wax. Prog. Lipid Res. 42, 51-80. doi: 10.1016/s0163-7827(02)00045-0

Lara, I., Belge, B., and Goulao, L. F. (2014). The fruit cuticle as a modulator of postharvest quality. Postharv. Biol. Tech. 87, 103-112. doi: 10.1016/j. postharvbio.2013.08.012 
Lara, I., Belge, B., Goulao, L. F. (2015). A focus on biosynthesis and composition of cuticle in fruits. JAFC 63, 4005-4019. doi: 10.1021/acs.jafc.5b00013

Lashbrooke, J., Adato, A., Lotan, O., Alkan, N., Tsimbalist, T., Rechav, K., et al. (2015a). The tomato MIXTA-Like transcription factor coordinates fruit epidermis conical cell development and cuticular lipid biosynthesis and assembly. Plant Physiol. 169, 2552-2571. doi: 10.1104/pp.15.01145

Lashbrooke, J., Aharoni, A., and Costa, F. (2015b). Genome investigation suggests MdSHN3, an APETALA2-domain transcription factor gene, to be a positive regulator of apple fruit cuticle formation and an inhibitor of russet development. J. Exp. Bot. 66, 6579-6589. doi: 10.1093/jxb/erv366

Lauter, N., Kampani, A., Carlson, S., Goebel, M., and Moose, S. P. (2005). microRNA172 down-regulates glossy 15 to promote vegetative phase change in maize. Proc. Natl. Acad. Sci. U.S.A. 102, 9412-9417. doi: 10.1073/pnas. 0503927102

Li, Z., Zhang, Z. H., Yan, P. C., Huang, S. W., Fei, Z. J., and Lin, K. (2011). RNA-Seq improves annotation of protein- coding genes in the cucumber genome. $B M C$ Genomics 12, 540. doi: 10.1186/1471-2164-12-540

Li, H. and Durbin, R. (2009) Fast and accurate short read alignment with Burrows-Wheeler transform. Bioinformatics 25: 1754-1760. doi: 10.1093/ bioinformatics/btp324

Love, M. I., Huber, W., and Anders, S. (2014). Moderated estimation of fold change and dispersion for RNA-seq data with DESeq2. Genome Biol. 15, 550-571. doi: 10.1186/S13059-014-0550-8

Lv, J., Qi, J., Shi, Q., Shen, D., Zhang, S., Shao, G., et al. (2012). Genetic diversity and population structure of cucumber (Cucumis sativus L.). PloS One 7, e46919. doi: 10.1371/journal.pone.0046919

Mansfeld, B. N., Colle, M., Kang, Y., Jones, A. D., and Grumet, R. (2017). Transcriptomic and metabolomic analyses of cucumber fruit peels reveal a developmental increase in terpenoid glycosides associated with age-related resistance to Phytophthora capsici. Hortic. Res. 4, 17022. doi: 10.1038/hortres.2017.22

Marçais, G., Delcher, A. L., Phillippy, A. M., Coston, R., Salzberg, S. L., and Zimin, A. (2018). MUMmer4: A fast and versatile genome alignment system. PloS Comput. Biol. 14 (1), e1005944. doi: 10.1371/journal.pcbi.1005944

Martin, L. B. B., and Rose, J. K. C. (2014). There's more than one way to skin a fruit: formation and functions of fruit cuticles. J. Exp. Bot. 65, 4639-4651. doi: $10.1093 /$ jxb/eru301

Matas, A. J., Agusti, J., Tadeo, F. R., Talon, M., and Rose, J. K. C. (2010). Tissuespecific transcriptome profiling of the citrus fruit epidermis and subepidermis using laser capture microdissection. J. Exp. Bot. 61, 3321-3330. doi: 10.1093/ jxb/erq153

Matas, A. J., Yeats, T. H., Buda, G. J., Zheng, Y., Chatterjee, S., Tohge, T., et al. (2011). Tissue- and cell-type specific transcriptome profiling of expanding tomato fruit provides insights into metabolic and regulatory specialization and cuticle formation. Plant Cell 23, 3983-3910. doi: 10.1105/tpc.111.091173

McKenna, A., Hanna, M., Banks, E., Sivachenko, A., Cibulskis, K., Kernytsky, A., et al. (2010). The genome analysis toolkit: A MapReduce framework for analyzing next-generation DNA sequencing data. Genome Res. 20, 1297-1303. doi: $10.1101 /$ gr.107524.110

Mintz-Oron, S., Mandel, T., Rogachev, I., Feldber, L., Lotan, O., Yativ, M., et al. (2008). Gene expression and metabolism in tomato fruit surface tissues. Plant Physiol. 147, 823-851. doi: 10.1104/pp.108.116004

Moose, S. P., and Sisco, P. H. (1996). Glossy15, an APETALA2-like gene from maize that regulates leaf epidermal cell identity. Genes Dev. 10, 3018-3027. doi: 10.1101/gad.10.23.3018

Nadakuduti, S. S., Pollard, M., Kosma, D. K., Allen, C.Jr., Ohlrogge, J. B., and Barry, C. S. (2012). Pleiotropic phenotypes of the sticky peel mutant provide new insight into the role of CUTIN DEFICIENT2 in epidermal cell function in tomato. Plant Physiol. 159, 945-960. doi: 10.1104/pp.112.198374

Nakano, T., Suzuki, K., Fujimura, T., and Shinshi, H. (2006). Genome-wide analysis of the ERF gene family in Arabidopsis and rice. Plant Physiol. 140, 411-432. doi: 10.1104/pp.105.073783

Oshima, Y., Shikata, M., Koyama, T., Ohtsubo, N., Mitsuda, N., and OhmeTakagi, M. (2013). MIXTA-like transcription factors and WAX INDUCER1/ SHINE1 coordinately regulate cuticle development in Arabidopsis and Torenia fournieri. Plant Cell. 25, 1609-1624. doi: 10.1105/tpc.113.110783

Patel, C., and Panigrahi, J. (2019). Starch glucose coating-induced postharvest shelf-life extension of cucumber. Food Chem. 288, 208-214. doi: 10.1016/j. foodchem.2019.02.123
Patro, R., Duggal, G., Love, M. I., Irizarry, R. A., and Kingsford, C. (2017). Salmon provides fast and bias-aware quantification of transcript expression. Nat. Methods 14, 417-419. doi: 10.1038/nmeth.4197

Petit, J., Bres, C., Just, D., Garcia, V., Mauxion, J. P., Marion, D., et al. (2014). Analyses of tomato fruit brightness mutants uncover both cutin-deficient and cutin-abundant mutants and a new hypomorphic allele of GDSL lipase. Plant Physiol. 164, 888-906. doi: 10.1104/pp.113.232645

Petit, J., Bres, C., Mauxion, J.-P., Bakan, B., and Rothan, C. (2017). Breeding for cuticle-associated traits in crop species: traits, target, and strategies. J. Exp. Bot. 68, 5369-5387. doi: 10.1093/jxb/erx341

Pyc, M., Cai, Y., Greer, M. S., Yurchenko, O., Chapman, K. D., Dyer, J. M., et al. (2017). Turning over a new leaf in lipid droplet biology. Trends Plant Sci. 22, 596-609. doi: 10.1016/j.tplants.2017.03.012

Qi, J., Liu, X., Shen, D., Miao, H., Xie, B., Li, X., et al. (2013). A genomic variation map provides insights into the genetic basis of cucumber domestication and diversity. Nat. Genet. 45, 1510-1515. doi: 10.1038/ng.2801

Rubenstein, M., Katzenellenbogen, M., Exhed, R., Rozen, A., Katzir, N., Colle, M., et al. (2015). Ultrahigh-density linkage map for cultivated cucumber (Cucumis sativus L.) using a single-nucleotide polymorphism genotyping array. PloS One 10, e0124101. doi: 10.1371/journal.pone.0124101

Sadre, R., Kuo, P., Chen, J., Yang, Y., Banerjee, A., Benning, C., et al. (2019). Cytosolic lipid droplets as engineered organelles for production and accumulation of terpenoid biomaterials in leaves. Nat. Commun. 10, 853. doi: 10.1038/s41467-019-08515-4

Shi, J. X., Adato, A., Alkan, N., He, Y., Lashbrooke, J., Matas, A. J., et al. (2013). The tomato SISHINE3 transcription factor regulates fruit cuticle formation and epidermal patterning. New Phytol. 197, 468-480. doi: 10.1111/nph.12032

Shimada, T. L., Hayashi, M., and Hara-Nishimura, I. (2018). Membrane dynamics and multiple functions of oil bodies in seeds and leaves. Plant Physiol. 176, 199-207. doi: 10.1104/pp.17.01522

Soneson, C., Love, M. I., Robinson, M. D. (2015). Differential analyses for RNAseq: transcript-level estimates improve gene-level inferences. F1000Research 4, 1521. doi: 10.12688/f1000research.7563.2

Taketa, S., Amano, S., Tsujino, Y., Sato, T., Saisho, D., Kadeda, K., et al. (2008). Barley grain with adhering hulls in controlled by an ERF family transcription factor gene regulating a lipid biosynthesis pathway. Proc. Nat. Acad. Sci. U.S.A. 105, 4062-4067. doi: 10.1073./pnas.0711034105

Taketa, S., Yuo, T., Yamashita, Y., Ozeki, M., Haruyama, N., Hidekazu, M., et al. (2012). "Molecular mechanisms for covered vs. naked caryopsis in barley. Adv. Barley Sci.," in Advance in Barley Sciences. Eds. Zhang, G., Li, C., and Liu, X. (Dordrecht: Springer). doi: 10.1007/978-94-007-4682-4_39

Trapnell, C., Williams, B. A., Pertea, G., Mortazavi, A., Kwan, G., van Baren, M. J., et al. (2010). Transcript assembly and quantification by RNA-Seq reveals unannotated transcripts and isoform switching during cell differentiation. Nat. Biotechnol. 28, 511-515. doi: 10.1038/nbt.1621

Trivedi, P., Nguyen, N., Hykkerud, A. L., Haggman, H., Martinussen, I., Jaakola, L., et al. (2019). Development and environmental regulation of cuticular wax biosynthesis in fleshy fruits. Front. Plant Sci. 10, 431. doi: 10.3389/ fpls.2019.00431

Van der Auwera, G. A., Carneiro, M. O., Hartl, C., Poplin, R., del Angel, G., LevyMoonshine, A., et al. (2013). "From FastQ data to high-confidence variant calls: The Genome Analysis Toolkit best practices pipeline," in Curr. Protoc. Bioinforma (Hoboken, NJ, USA: John Wiley \& Sons, Inc.), 11.10.1-11.10.33.

Wang, S., Basten, C. J., and Zeng, Z.-B. (2012). Windows QTL Cartographer 2.5. Raleigh, NC: Department of Statistics, North Carolina State University. http:// statgen.ncsu.edu/qtlcart/WQTLCart.htm.

Wang, W. Liu, X., Gai, X., Ren, J., Liu, X., Cai, Y., Wang, Q., and Ren, R. (2015a). Cucumis sativus L. WAX2 plays a pivotal role in wax biosynthesis, influencing pollen fertility and plant biotic and abiotic stress responses. Plant Cell Physiol. 56, 1339-1354. doi: 10.1093/pcp/pcv052

Wang, W. J., Zhang, Y., Xu, C., Ren, J. J., Liu, X. F., Black, K., et al. (2015b). Cucumber ECERIFERUM1 (CSCER1), which influences the cuticle properties and drought tolerance of cucumber, plays a key role in VLC alkanes biosynthesis. Plant Molec. Biol. 87, 219-233. doi: 10.1007/s11103-014-0271-0

Wang, X., Bao, K., Reddy, U. K., Bai, Y., Hammar, S. A., Jiao, C., et al. (2018). The USDA cucumber (Cucumis sativus L.) collection: genetic diversity, population structure, genome-wide association studies and core collection development. Hortic. Res. 5, 64. doi: 10.1038/s41438-018-0080-8 
Wei, G., Tian, P., Zhang, F., Qin, Q., Miao, H., Chen, Q., et al. (2016). Integrative analysis of nontargeted volatile profiling and transcriptome data provide molecular insight into VOC diversity in cucumber plants (Cucumis sativus). Plant Physiol. 172, 603-618. doi: 10.1104/pp.16.01051

Weng, Y., Colle, M., Wang, Y. H., Yang, L. M., Rubinstein, M., Sherman, A., et al. (2015). QTL mapping in multiple populations and development stages reveals dynamic quantitative trait loci for fruit size in cucumbers of different market classes. Theor. Appl. Genet. 128, 1747-1763. doi: 10.1007/ s00122-015-2544-7

Wiersma, A. T., Pulman, J. A., Brown, L. K., Cowger, C., and Olson, E. L. (2017). Identification of Pm58 from Aegilops tauschii. Theor. Appl. Genet. 130, 11231133. doi: 10.1007/s00122-017-2874-8

Yang, L., Koo, D. H., Li, Y., Zhang, X., Luan, F., Havey, M. J., et al. (2012). Chromosome rearrangements during domestication of cucumber as revealed by high-density genetic mapping and draft genome assembly. Plant J. 71, 895906. doi: 10.1111/j.1365-313X.2012.05017

Yang, X. Q., Yue, L., Zhang, W. W., He, H. L., Pan, J. S., and Cai, R. (2014a). Fine mapping of the uniform immature fruit color gene $\mathrm{u}$ in cucumber (Cucumis sativus L.). Euphytica. 196, 341-348. doi: 10.1007/s10681-013-1037-5

Yang, X., Zhang, W., Li, Y., He, H., Bie, B., Ren, G., et al. (2014b). mapping of the dull fruit skin gene D in cucumber (Cucumis sativus L). Mol. Breed. 33, 15-22. doi: 10.1007/s11032-013-9927-8

Yang, X., Zhang, W., He, H., Nie, J., Bie, B., Zhao, J., et al. (2014c). Tuberculate fruit gene $\mathrm{Tu}$ encodes a $\mathrm{C} 2 \mathrm{H} 2$ zinc finger protein that is required for the warty fruit phenotype in cucumber (Cucumis sativus L.). Plant J. 78, 1034-1046. doi: $10.1111 /$ tpj.12531
Yang, S., Wen, C., Liu, B., Cai, Y., Xue, S., Bartholomew, E. Z., et al. (2019). A $\mathrm{CsTu}$-Ts1 regulatory module promotes fruit tubercule formation in cucumber. Plant Biotech. J. 17, 289-301. doi: 10.1111/pbi.12977

Yeats, T. H., and Rose, J. K. (2013). The formation and function of plant cuticles. Plant Physiol. 163, 5-20. doi: 10.1104/pp.113.222737

Yeats, T. H., Martin, L. B., Viart, H. M., Isaacson, T., He, Y., Zhao, L., et al. (2012). The identification of cutin synthase: formation of the plant polyester cutin. Nat. Chem. Biol. 8, 609-611. doi: 10.1038/nchembio.960

Zhang, S., Miao, H., Song, Z. C., Liu, P. N., Wang, Y., Wehner, T. C., et al. (2017) Molecular mapping and candidate gene analysis for fruit epidermal structure in cucumber. Plant Breed. 136, 767-774. doi: 10.1111/pbr.12510

Zheng, Y., Wu, S., Bai, Y., Sun, H., Jiao, C., Guo, S., et al. (2019). Cucurbit Genomics Database (CuGenDB): a central portal for comparative and functional genomics of cucurbit crops. Nucl. Acids Res. 47, D1128-D1136. doi: 10.1093/nar/gky944

Conflict of Interest: The authors declare that the research was conducted in the absence of any commercial or financial relationships that could be construed as a potential conflict of interest.

Copyright (C) 2019 Rett-Cadman, Colle, Mansfeld, Barry, Wang, Weng, Gao, Fei and Grumet. This is an open-access article distributed under the terms of the Creative Commons Attribution License (CC BY). The use, distribution or reproduction in other forums is permitted, provided the original author(s) and the copyright owner(s) are credited and that the original publication in this journal is cited, in accordance with accepted academic practice. No use, distribution or reproduction is permitted which does not comply with these terms. 